\title{
Relaxation towards localized vorticity states in drift plasma and geostrophic flows
}

\author{
Olivier Agullo* \\ Équipe Dynamique des Systèmes Complexes, PIIM, \\ Centre universitaire de Saint-Jérôme, F-13397 Marseille Cedex 20, France \\ Alberto Verga $^{\dagger}$ \\ Institut de Recherche sur les Phénomènes Hors Équilibre, \\ 49, rue F. Joliot-Curie, BP 146, 13384 Marseille, France
}

(Dated: September 7, 2006)

\begin{abstract}
The drift of ions in a magnetized plasma or the height fluctuations of a rotating fluid layer are described by the conservation equation of a potential vorticity. This potential vorticity contains an intrinsic length scale, the hybrid Larmor radius in plasma, and the Rossby length in the quasigeostrophic flow. The influence of this scale in the evolution of a random initial vorticity field is investigated using a thermodynamic approach. In contrast to the perfect fluid case, where the vorticity tends to a well defined stationary state, complete relaxation towards an equilibrium state is not observed in numerical simulations of quasi-geostrophic decaying turbulence. The absence of global thermodynamic equilibrium is explained by the relaxation towards states of local equilibrium where the vorticity is concentrated. Interaction between these separated regions is extremely weak. Explicit, axisymmetric, localized solutions of the mean field integro-differential equation of extremal entropy states are obtained using asymptotic methods. Comparison of the computed solutions with the observed coherent structures, shows that they effectively correspond to states in local thermodynamic equilibrium.
\end{abstract}

PACS numbers: $47.52 .+\mathrm{j}, 47.27 . \mathrm{Qb}, 05.40 \mathrm{j}$

\section{INTRODUCTION}

A class of plasmas and fluid flows can be described, at least approximately, by the conservation of a potential vorticity. This is the case of the drift motion of ions in a magnetized plasma, or of the flow of a rotating fluid layer sometimes called quasi-geostrophic flow. For such systems the potential vorticity satisfies a two-dimensional Euler equation, expressing the conservation of the potential vorticity along the streamlines. The relation between potential vorticity $\Omega$ and stream function $\psi$ is given by

$$
\Omega=-\Delta \psi+\psi / l^{2},
$$

where $l$ is some intrinsic length, and $\Delta$ is the twodimensional Laplacian. The evolution equation for the potential vorticity is then

$$
\frac{\partial \Omega}{\partial t}+[\Omega, \psi]=0
$$

where $t$ is the time, and $[\cdot, \cdot]$ is the Poisson bracket.

In order to clearly state the physical nature of (2), and the origin of the intrinsic length, let us consider first, the slow motion of ions in a homogeneous, magnetized plasma. The uniform magnetic field is supposed to be along the $z$-direction, $\boldsymbol{B}=B \hat{\boldsymbol{z}}$, and all physical quantities depend on the $(x, y)$-coordinates. We assume the

\footnotetext{
*Electronic address: agullo@up.univ-mrs.fr; UMR 6633, CNRS, Université de Provence

${ }^{\dagger}$ Electronic address: Alberto.Verga@irphe.univ-mrs.fr; UMR
} 6594, CNRS, Université de Provence plasma quasi-neutral, and the electrons adiabatic. The density of ions $n$ can then be related to the electrostatic potential $\phi$ by the Boltzmann law

$$
n=n_{0} e^{e \phi / T_{e}},
$$

where $n_{0}$ stands for the equilibrium plasma density, $T_{e}$ for the electron temperature (in energy units), and $e$ for the electron charge. Using this expression for $n$, the ion continuity equation is given by

$$
\boldsymbol{\nabla} \cdot \boldsymbol{v}=-\frac{D}{D t}\left(\frac{e \phi}{T_{e}}\right),
$$

where $\boldsymbol{v}$ is the ion velocity, and $D / D t$ is the total derivative $\partial / \partial t+\boldsymbol{v} \cdot \boldsymbol{\nabla}$. The motion equation is

$$
\frac{D}{D t} \boldsymbol{v}=-\frac{e}{m_{i}} \nabla \phi+\omega_{c} \boldsymbol{v} \times \hat{\boldsymbol{z}},
$$

where we neglected the ion pressure with respect to the Lorentz force, the right hand side of (4) (we consider a plasma with the electron temperature much larger than the ion one). In this equation $m_{i}$ is the ion mass, and $\omega_{c}=e B / m_{i}$ the cyclotron frequency.

Before proceeding, it is interesting to note that (3) and (4) are similar to the hydrodynamic equations describing the motion of a rotating fluid layer of height $H=H_{0}+h$ ( $H_{0}$ is the mean width of the layer), in which the pressure is proportional to the variations of the layer width $h(x, y, t)$. We assume, as in the shallow water approximation, that the fluid layer is thin with respect to the characteristic scales of the horizontal motion. We may identify the height variations $h$ with the electrostatic potential, and the magnetic force with the Coriolis force 
(we neglect variations of the rotation frequency, the socalled "beta" effect in geostrophic flows). In such a case we would have

$$
\frac{\partial H}{\partial t}+\nabla \cdot(H \boldsymbol{v})=0
$$

or equivalently,

$$
\boldsymbol{\nabla} \cdot \boldsymbol{v}=-\frac{D}{D t} \log \left(1+\frac{h}{H_{0}}\right)
$$

and

$$
\frac{D}{D t} \boldsymbol{v}=-g \nabla h+f \boldsymbol{v} \times \hat{z}
$$

where $g$ is the acceleration due to gravity, and $f / 2$ is the rotation frequency. These equations, which describe the quasi-geostrophic flow, are formally equivalent to the plasma equations (3-4).

In the plasma case we can form a characteristic length from the so-called hybrid sound speed $c_{s}^{2}=T_{e} / m_{i}$ appearing in the electrostatic force term, and the cyclotron frequency, to obtain the hybrid Larmor radius $l_{L}=c_{s} / \omega_{c}$. In the fluid case, the characteristic speed is $c^{2}=g H_{0}$, the characteristic length is $l_{R}=c / f$, the Rossby length ( $f$ is in this case the characteristic frequency). The plasma and fluid systems are thus equivalent provided we identify $e \phi / T_{e}$ and $h / H_{0}$ and take length and time units $\left(l_{L}, 1 / \omega_{c}\right)$ or $\left(l_{R}, 1 / f\right)$ respectively.

As our objective is to obtain an equation for the vorticity $\omega=(\boldsymbol{\nabla} \times \boldsymbol{v}) \cdot \hat{\boldsymbol{z}}$ (only the $z$-component of the vorticity is not zero, in these two-dimensional systems), we compute the rotational of (4):

$$
\boldsymbol{\nabla} \cdot \boldsymbol{v}=-\frac{1}{\omega+\omega_{c}} \frac{D}{D t} \omega .
$$

Since we are interested in the slow motion of ions (or the slow variations of the layer width), we assume that the temporal variations of the electrostatic fluctuations is much smaller than the cyclotron frequency $|\partial / \partial t| \ll \omega_{c}$, which means that in the first approximation the vorticity is given by the drift velocity

$$
\omega \approx-\nabla \times(\nabla \phi \times \hat{z} / B) \cdot \hat{z},
$$

and then,

$$
\omega=\omega_{c} l_{L}^{2} \Delta\left(e \phi / T_{e}\right) \ll \omega_{c} .
$$

In the case of the geostrophic flows, the equivalent approximation is that of a small Rossby number $\omega / \omega_{R}$. Therefore, we can neglect $\omega$ with respect to $\omega_{c}$ in (8), and combine it with (3), to finally obtain the conservation equation,

$$
\frac{D}{D t}\left(\Delta \phi-\phi / l_{L}^{2}\right)=0
$$

which can easily be transformed to the generalized Euler equation (2), by identifying the electrostatic potential with the stream function $\psi=-\omega_{c} l_{L}^{2} e \phi / T_{e}$, or $\psi=$ $-f c^{2} h / H_{0}$ in the fluid case, and with the vorticity given by $\omega=-\Delta \psi$ in both cases.

Interest in this type of equations started with the study of atmospheric motion in the geostrophic approximation, and later in plasmas [17], and Jupiter's red spot [26]. A systematic comparison of both flows was made by Hasegawa, Maclennan and Kodama [18]; more recently Pésceli and Trulsen [35] investigated some of their statistical properties, and Watanabe, Iwayama and Fujisaka [47] performed numerical simulations of the evolution of an initially random state. The more general case of the shallow water system, a compressible fluid layer in a rotating frame, was studied by Chavanis and Sommeria [10]. Hopfinger and van Heijst [19] surveyed several topics on vortices in rotating fluids. A review on the analogy between drift plasma and geophysical flows, including several generalizations, in particular on nonlinear effects, can be found in [32].

One important feature of these systems is that a superposition of point vortices, sometimes called screened vortices or Stewart vortices, are exact solutions of (2), in much the same way as for the Euler case [42], [33]. At variance to the usual Euler $\log (r)$ interaction $(r$ is the distance between two point vortices), geostrophic point vortices of (2) interact with $\mathrm{K}_{0}(r / l)$ law, where $\mathrm{K}_{0}$ is the modified Bessel function. (Although "Euler" and "geostrophic" are not fully appropriated denominations, we use them for simplicity.) We remark that for an Euler flow there is no characteristic length, other than those related to initial conditions. The change $r \rightarrow \alpha r$, with $\alpha$ an arbitrary constant, lets invariant the equation of motion of point vortices, and also Euler equation, after an appropriated change in the time units. On the other hand, the interaction of point vortices in the geostrophic flow involves the characteristic length $l$, and obviously the motion of vortices explicitly depends on this scale. Systems of Euler and geostrophic point vortices might then behave differently, as usually happens between long and short range interacting particle systems.

\section{A. Statistical mechanics of turbulence}

The possibility of approximating the vorticity flow by a superposition of point vortices, led to the idea that two-dimensional turbulence can be described using the usual statistical methods for Hamiltonian systems. In 1949, Onsager [34] settled the bases of a thermodynamic approach to hydrodynamics. He demonstrated in particular that the phase space of a system of point vortices has finite volume and consequently states of negative temperature are thermodynamically accessible. Negative temperature states are characterized by the formation of clusters of like circulation vortices. In the Euler case, as the interaction range is infinite, this process continues up to the formation of vortices whose size is comparable to the size of the system, and a global equilibrium state is predicted. An analogous thermodynamical approach is valid 
for the quasi-geostrophic flow, with $\Omega$ replaced by $\omega$, and therefore, this global state should also be reached in this case (this is the generally accepted viewpoint, see reference [10]). However, and this is the subject of the present paper, due to the short range interaction of geostrophic vortices, localized distribution of vorticity may develop (as is the case for the Hasegawa-Mima system, [12]). In such a case distant vortices will interact weakly, leading to a very slow relaxation towards equilibrium, slower than any relevant time, as for instance, viscous time, and the appropriate asymptotic state of the system will be not longer a global but a local thermodynamic state.

The theory of Onsager was extensively studied, first to systematically derive the relevant thermodynamic quantities as the free energy, using microcanonical or canonical approaches to the point vortex systems [23], [36], [24], [2], and later to incorporate into the statistical formalism the continuous distribution of vorticity and the infinite number of invariants other than energy and enstrophy [37], [30].

One important result of these works is that the most probable state (which is an extremum of the free energy) is given by a Debye like equation for an electrolyte, relating the vorticity to the current function:

$$
\Omega=\alpha_{+} e^{-\beta \Gamma \psi}-\alpha_{-} e^{\beta \Gamma \psi},
$$

where $\beta$ is a parameter, like a Lagrange multiplier, equivalent to the inverse of a temperature (note however, that $\beta$ has the dimensions of an area $\mathcal{A}$ over an energy); $\alpha_{ \pm}$ are two constants which take into account that the integral of (10) over the entire fluid domain $\mathcal{A}$, should vanish. A very simple explanation of this formula is given by the analogy of the vortex system, which satisfy the Poisson equation

$$
-\Delta \psi=\Omega-\psi / l^{2}=\omega(\psi),
$$

and a dilute electrolyte. The vorticity $\omega$ is equivalent to the charge density, and the stream function to the mean field electrostatic potential. The equilibrium distribution of vortices (charges, in this analogy), is the Boltzmann distribution, proportional to $\exp (\beta \Gamma \psi)$, giving directly an equation of the type of (10). In fact the analogy of the vortex system with a system of Coulomb interacting particles is rather misleading: the "temperature" of vortices can take both signs [34], and the negative temperature states are the most relevant in hydrodynamics. Another analogy which was sometimes exploited in the literature is to compare the (point) vortex systems with a stellar system, interacting with the gravitational potential (see e.g. [6]). However, in addition that for stars only attractive interactions are allowed, the Hamiltonian possess both kinetic and potential energies, and then the phase space is not bounded as it is in the case of a vortex system.

The integro-differential equation (10) establishes a functional relation $\Omega=\Omega(\psi)$. Clearly, its solution is also a stationary exact solution of $(2)$ because the Poisson bracket vanishes identically. If the usual hypothesis on the validity of statistical thermodynamics are satisfied, the system evolves from an arbitrary initial state to a stationary state with precisely this distribution of vorticity. However, one important difference might exist in the nature of the equilibrium states, between rotation dominated flows and Euler flows. This difference would be related to the intrinsic length, which adds a term $\psi / l^{2}$ to the potential vorticity. Indeed, suppose that $\alpha_{-}=\alpha_{+}=\alpha / 2$ (this is not essential to the present argument), and then $\omega=\omega(\psi)$ in equation (11) is given by

$$
\omega(\psi)=-\psi / l^{2}-\alpha \sinh (\beta \Gamma \psi)
$$

We note that due to the first term in the right hand side, $\omega(\psi)$ can change its sign near the origin ( $\psi$ small enough) for negative temperature, $\beta<0$. This can be shown by a simple analogy with the one-dimensional motion of a particle in a potential $\mathcal{V},\left(\Delta=d^{2} / d x^{2}\right.$ becomes the acceleration of the particle, if we interpret the coordinate $x$ as the time)

$$
\begin{gathered}
d^{2} \psi / d x^{2}=\omega=-d \mathcal{V}(\psi) / d \psi, \\
\mathcal{V}=-\psi^{2} / 2 l^{2}-\alpha \cosh (\beta \Gamma \psi) / \beta \Gamma .
\end{gathered}
$$

This equation allows us to see that two cases arise: when $\beta>0$ only the solution $\psi=0$ exists; and, when $\beta<0$ two other solutions appear. This change in sign, implies then the existence of two local minima, separated by a separatrix. The motion on the separatrix is localized in space, it approaches zero exponentially fast in both directions, the "period" of the motion becomes infinite.

Therefore, the presence of an intrinsic length opens the possibility of localized distributions of vorticity at local equilibrium, weakly interacting with other structures. The essential ingredient is the short range interaction associated with this length. The existence of such regions may also be related to the fact that the area of vortices generally diminishes after fusion [5], the distance between vortices tends then to increase. Let us assume that the size of the system is $L$ much greater than the interaction length, and introduce an intermediate scale $l_{B}$ such that the system can be divided into many boxes of size $l_{B}$, $l \ll l_{B} \lesssim L$. (Note that this hierarchy of scales cannot in general be introduced in Euler flows; we discuss this point later on $\S \mathrm{V}$.) If the boxes are large enough and interactions between two boxes are negligible (confined to a layer of size $l$ ), it is natural to think that they should evolve to different and independent thermodynamic states, relaxation to an equilibrium (statistical stationary state) being faster than mixing of distant boxes.

In $\S$ II we investigate the phenomenology of this system to test the scenario of slow relaxation. A brief account of the thermodynamical formalism is given in $\S$ III, followed by $\S \mathrm{IV}$, where we compute explicit solutions of the thermodynamic equation. A comparison is also made between the (almost) stationary vortices found in the 
numerical simulations and the solutions of the thermodynamic equations. Our main conclusion, presented in $\S \mathrm{V}$, is that in contrast to the decaying two-dimensional Navier-Stokes "turbulence", for which the system attains a well defined thermodynamic state (see however, the discussions in [39], and [48]), the geostrophic decaying turbulence evolves towards a peculiar turbulent state, characterized by a superposition of coherent vortices in local thermodynamic equilibrium.

\section{NUMERICAL SIMULATIONS OF DECAYING TURBULENCE AND RELAXATION}

The pioneering experiments of Couder [11] showed that two-dimensional turbulence is dominated by the interaction of coherent vortices. In these experiments, turbulence was generated on a thin soap film flowing through a grid. The system freely evolved by successive merging of vortices, in such a way that the mean size of vortices grew with time. The long time behavior of almost inviscid of two-dimensional turbulent flow was investigated by Fine et al. [13], using an electron plasma column. They obtained a final stationary state consisting in a few vortices forming a quasi-crystal structure. Most of the experimental studies in laboratory concern Euler turbulence, the long time evolution of decaying quasigeostrophic turbulence is less known. Therefore, it is interesting to perform direct numerical simulations that can reproduce some phenomenological features of the turbulence and relaxation in the quasi-geostrophic case.

Two-dimensional Euler turbulence was intensively investigated by numerical simulations. The early stages of decaying turbulence, and the formation of coherent structures, were studied by McWilliams et al. [28], [29], and an interpretation of the observed decaying laws, is given by Carnevale et al. [5], and Weiss and McWilliams [46]; other recent numerical results may be found in $[1,4]$. For a study of the late stages, and for a description of the asymptotic stationary states in terms of the thermodynamic theory, see e.g. [27], [31], [3], and [39]. Watanabe, Iwayama and Fujisaka [47] recently performed simulations of the geostrophic flow but they were only concerned with the initial transitory regime of decaying turbulence and the dynamics of coherent structures.

In fact, numerically one does not integrate Euler equations but instead, some dissipative version of these equations. The most natural choice is Navier-Stokes, with a normal viscosity term $\nu \Delta \Omega(\nu$ is the kinematic viscosity coefficient) added to (2), but often some hyperviscosity is used (see the paper by Jiménez [20], for a discussion about the physical consequences of the hyperviscosity on the properties of vortices).

We performed a series of moderate resolution (number of grid points $N^{2}=256^{2}$ ), pseudo-spectral numerical integrations of (1) and (2), with a viscous term $(\nu=$ $10^{-3}$, in most cases). The code uses a predictor-corrector method which exactly updates linear terms (see appendix

$\begin{array}{lccccccc}\text { Name } & \nu & l & E_{0} & O_{0} & \text { time } & \text { IC } & \text { fig. } \\ \text { K0 } & 10^{-3} & \infty & 0.015 & 0.02 & 300 & 1 & 5 \\ \text { K10 } & 10^{-3} & 1 / 3 & 0.015 & 0.25 & 600 & 2 & 3 \\ \text { K36 } & 10^{-3} & 1 / 6 & 0.025 & 1 & 900 & 2 & 3,5 \\ \text { LI } & 10^{-3} & 1 / 6 & 0.25 & 10 & 600 & 1 & 1,4,5 \\ \text { LI1 } & 10^{-3} & 1 / 6 & 0.5 & 20 & 1100 & 1 & 4,5 \\ \text { LI3 } & 210^{-4} & 1 / 3 & 0.1 & 2 & 50 & 1 & 2\end{array}$

TABLE I: Simulation parameters. IC stand for the type of initial conditions. Type 1 is a random distribution of vorticity. Type 2 consists of a superposition of a few Fourier modes. The last column refers to the figure number.

$\S A)$. In table I we summarize the parameters used in the simulations. We varied the value of the intrinsic length $l$, and for a given $l$, we changed initial conditions to obtain different energies $E_{0}=E(0)$, and enstrophies $O_{0}=O(0)$,

$$
\begin{gathered}
E(t)=\frac{1}{2 \mathcal{A}} \int_{\mathcal{A}} d x d y\left[(\nabla \psi)^{2}+\frac{\psi^{2}}{l^{2}}\right], \\
O(t)=\frac{1}{2 \mathcal{A}} \int_{\mathcal{A}} d x d y \Omega^{2} .
\end{gathered}
$$

The area of the square box is $\mathcal{A}=(2 \pi)^{2}$, and length units are then given in terms of the box size $L=2 \pi$. Time is measured in (arbitrary) dimensional units: this initial value problem has not a preferred (intrinsic) time characteristic scale. The viscous time scale is for example, $\tau_{\nu}=1 / \nu \approx 10^{3}$ for structures of the box size, typical vortex turnover times are $\tau_{\omega}=1 / \sqrt{O_{0}} \approx 1$.

These integral quantities, $E$ and $O$, depend on time due to the viscosity. We used two types of vorticity distribution to start the simulations. The first one has a few Fourier modes, and is called "ordered". The second one, has a wide spectrum, and is called "random". In the second case the initial stream function is

$$
\psi(x, y)=\operatorname{Re} \sum_{n, m=-N / 2}^{N / 2} a_{n, m} e^{i(n x+m y)+i \phi_{n, m}},
$$

where the power spectrum decays as $\left|a_{n, m}\right|^{2} \sim k^{-6}$ for large wavenumber $k^{2}=n^{2}+m^{2}$, and the phases $\phi_{n, m}$ are random, uniformly distributed on the circle. The "ordered" initial condition used wavenumbers in the range $(1,7)$. In this paper, we do not focus on the energy power spectrum evolution and the correlated problem of the self-similarity breaking in turbulent decay [22], [38]. However, this initial spectrum has been chosen in order to have initial conditions close of those of McWilliams [28] and also Matthaeus et al. [27], which allows some comparisons with previous works on Euler flows.

In figure 1 we show the evolution of $E$ and $O$, and note that their decay is rather slow, in particular the energy varied only a few percent, between $t=300$ and $t=600$, while the state of the system remained, as we will show, almost unchanged. 


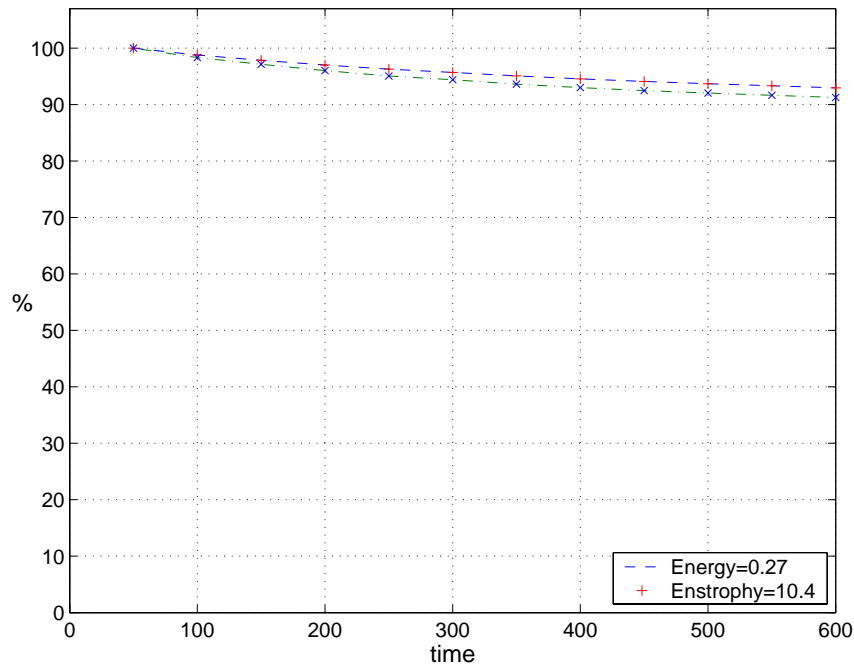

FIG. 1: Temporal evolution of the energy $(+)$ and the enstrophy $(\times), l=1 / 3$. Initial values are $E(0)=0.27$ and $O(0)=10.4$.

The initial evolution of the system is similar to the Euler case. The vorticity concentrates in large vortices through various generations of fusion processes. In figure 2 we show the distribution of the vorticity at different times, in the case with $l=1 / 3$ and with initial energy $E(0)=0.1$ (simulation LI). The initial random field selforganize in localized structures, the further evolution of the system depends on the interactions of these vortices. In the Euler case, although different configurations were observed, the flow tends to a steady state of thermodynamic equilibrium (provided that the system is mixing, as we discuss in $\S \mathrm{V})$. For this kind of initial conditions, the typical state is characterized by a functional relation of the form $\omega \sim \sinh \psi,[27]$.

The quantity $(E / O)^{1 / 2}=l_{E}$ has dimensions of a length, and one can ask whether this "integral" length is related to the long time structures emerging from the initial random state. We performed simulations with different values of this ratio, $L / l_{E} \approx 20,40$, but the evolution of the system is mainly influenced by the actual value of the total energy. Simulations K36, LI and LI1, have the same ratio $E / O$, and evolved to completely different states, with vortex sizes not directly related to $l_{E}$. However, a more systematic investigation of the decay processes, and the influence of initial conditions, is necessary to answer this question, which is out of the scope of the present work.

At variance to the Euler case with equivalent initial conditions, where a well defined equilibrium state set in, the geostrophic system slowly evolves to a quasistationary state, characterized by the presence of a few localized vortices. In figures 3 and 4 we show the late evolution of the flow for various simulation parameters.

We find that in spite of the very long simulation time, and for the various random initial conditions we used, the system does not reach an equilibrium state. The quasi-stationary state observed is of another nature, in the sense that no further merging nor new generation of vortices are observed. This state is characterized by the presence of localized vortices, which slowly move and evolve around a field of small fluctuations. We also see in the simulations that these coherent structures are separated by strong vorticity gradients, which may have a characteristic scale length of the same order as the interaction length $l$. Here we define loosely a coherent structure as an isolated, long lived vortex; more sophisticated definitions abound in the literature [16]. One property of these distinct regions is that their average vorticity is different from zero (the value of the global mean vorticity). The presence of such regions may be related to the dynamical segregation, by like sign vortex merging, of positive and negative dominated structures. We shall see in section $\S \mathrm{IV}$ that this state corresponds to a local thermodynamical equilibrium. A phenomenological indication that this can be the case is illustrated by the fact that in the large vortex regions, vorticity and stream function are related, and then they are near to a stationary solution.

In figure 5 we show the functional relation relating the vorticity to the stream function. We retrieve in the Euler case the well known result that the flow tends to a state where $\omega \sim \sinh \psi$. The simulations of the geostrophic flow show radically different behaviours. In the simulation K36 we found that the quasi-stationary state is characterized by the superposition of two functions $\omega(\psi)$. On the other hand, we clearly see in both K36 and LI, the distinction between the coherent vortices and the low amplitude fluctuations. The LI long time behaviour is remarkable, the high amplitude positive vortex does not has its negative counterpart, even if the mean value of the stream function is zero (and conserved by the dynamics). In fact, this asymmetry between positive and negative vortices is observed in all simulations with finite $l$. For instance, the four vortices of K36 have different shapes and amplitudes.

Another important fact, as we can see in the LI1 simulation, is that at very long times $(t=1100)$ a nice $\omega(\psi)$ function is established, as for the Euler flow, but with a negative slope at the origin (see figure 5). This behaviour agrees with the functional form one expects in a state of thermodynamic equilibrium, as we anticipated in the Introduction, equation (12).

The evolution of the quasi-geostrophic flows observed in the simulations supports the idea of a very slow relaxation to a global thermodynamical equilibrium state. After the initial decaying turbulence regime, where the system is dominated by the merging of vortices processes, a quasi-equilibrium state establishes. It is characterized by the coexistence of small amplitude fluctuations, and slowly moving large vortices. The large vortices appear to be close to equilibrium, with a well defined $\omega(\psi)$ relation. As their interaction with the background fluctuations is very weak, this regime lasts for long times, in our simulations to times comparable to the large scale 

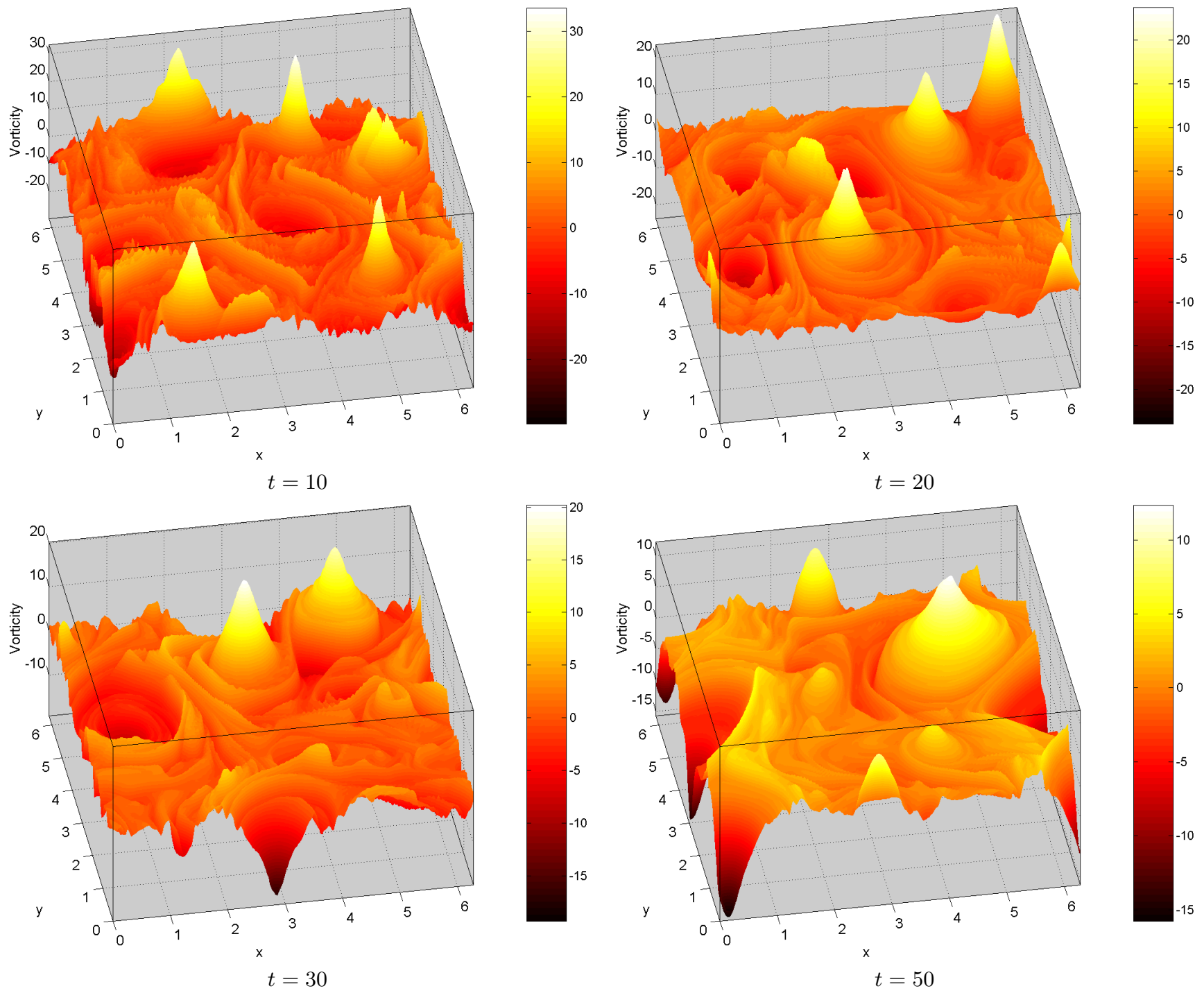

FIG. 2: Surface plots of the vorticity at times $t=10,20,30,50$ (simulation LI3).

viscous time. This behaviour strongly differs from the one prevailing in Euler flow, where the system globally evolves to a thermodynamic state, in times much smaller than the viscous one (under similar initial and boundary conditions). These results justify the application of a thermodynamic approach to describe the formation and stability of localized vortices.

The existence of a quasi-stationary state, where localized coherent structures dominate the flow, were also observed in electrostatic plasma turbulence [12]. The role of small scale fluctuations was enhanced in this case because of the presence of propagating dispersive drift waves. A similar situation, with Rossby waves, can be found in geostrophic flows.

\section{THERMODYNAMIC FORMALISM AND MEAN FIELD VORTICITY EQUATION}

We briefly review in this section the derivation of the vorticity equation for a local thermodynamic equilibrium state. Although formally the derivation follows the well known procedure $[23,24,30]$, in the present case the framework is different: we consider a sub-domain of the flow, denoted by $\mathcal{D}$, large enough to be described by a large set of point vortices, weakly interacting with the rest of the system; the system itself consisting in a superposition of these subsystems. An immediate consequence of this hypothesis is that the natural thermodynamical ensemble is the canonical one, the weak interaction between sub-domains leading to fluctuations in their total energy. Another consequence is that the validity of the thermodynamical approach is limited to an intermediate time range: long enough to mix the initial vorticity, but 
K10
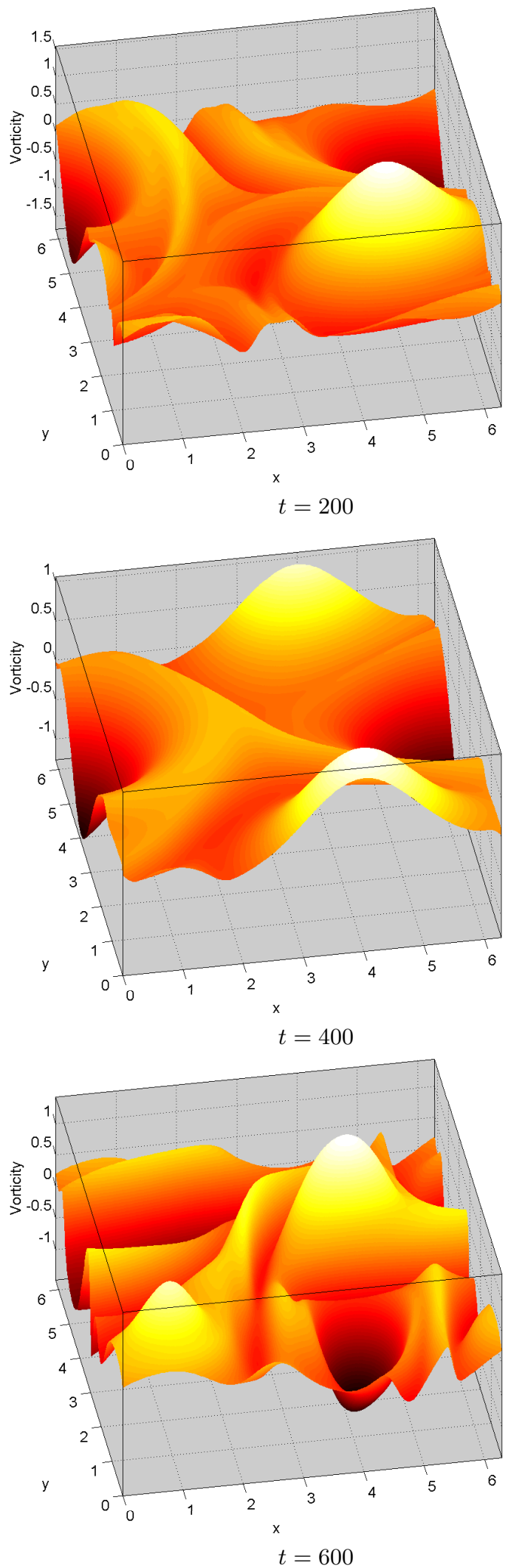

K36
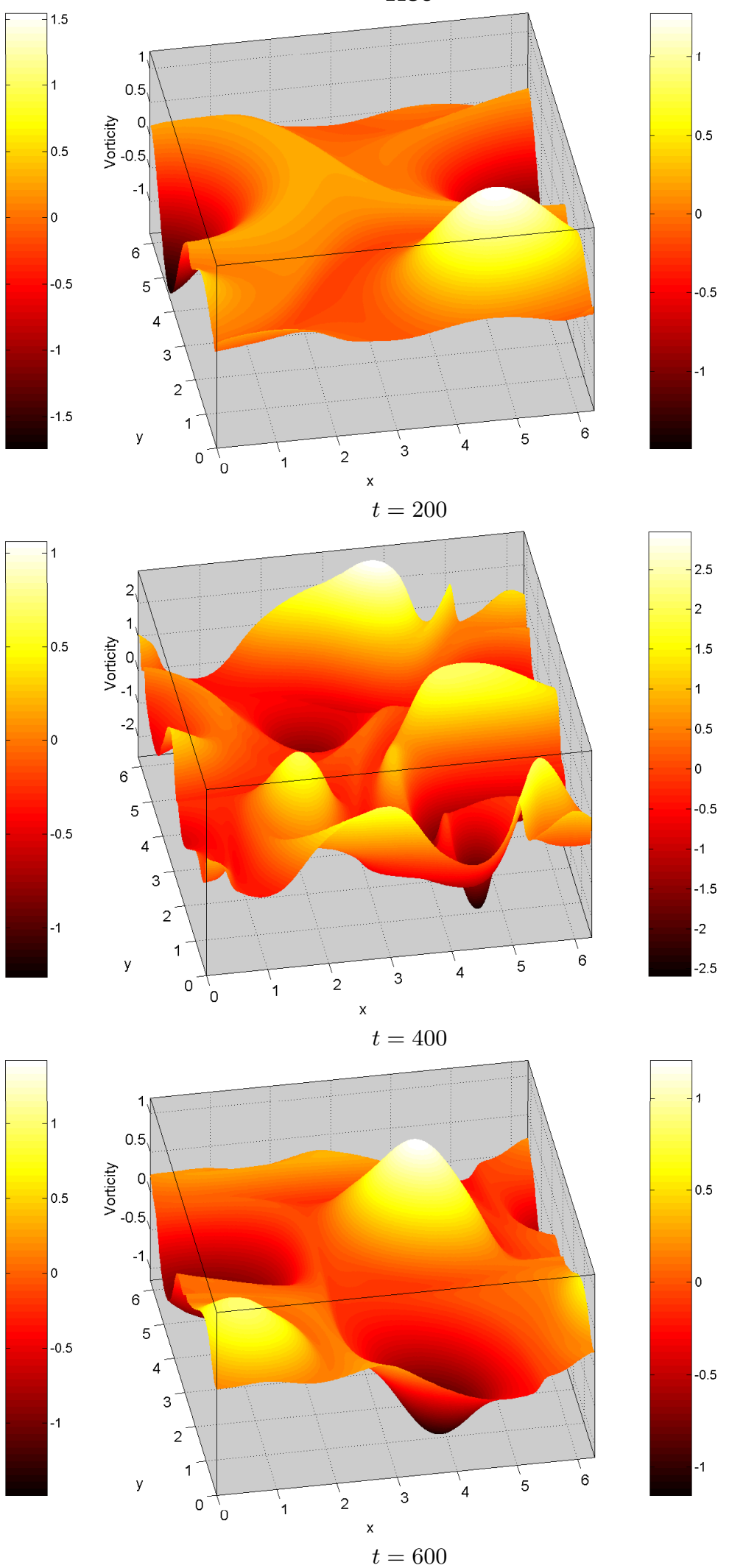

FIG. 3: Late stage of the evolution. Surface plots of the vorticity at times $t=200,400,600$ (simulations K10 and K36). 

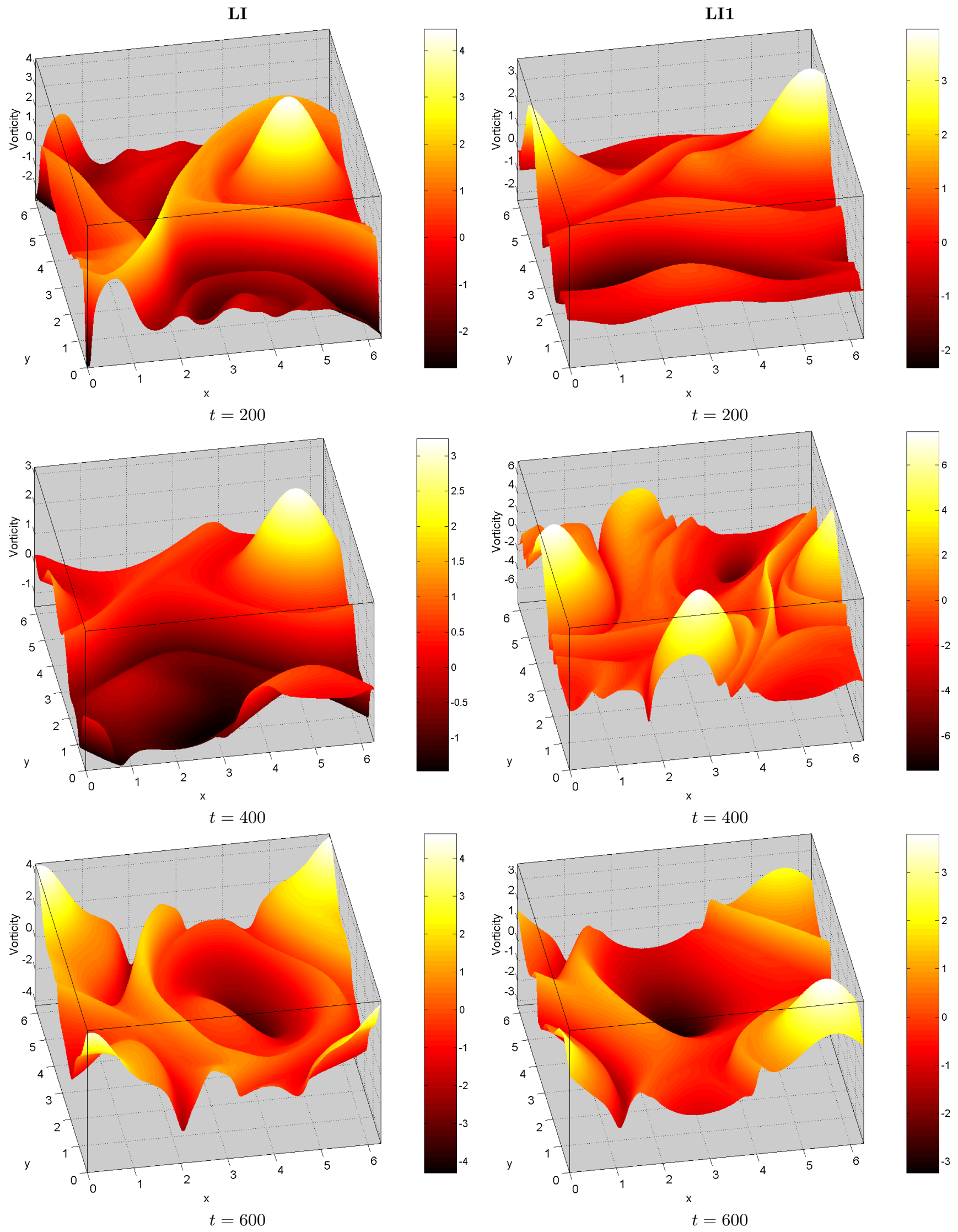

FIG. 4: Late stage of the evolution. Surface plots of the vorticity at times $t=200,400,600$ (simulations LI and LI1). 

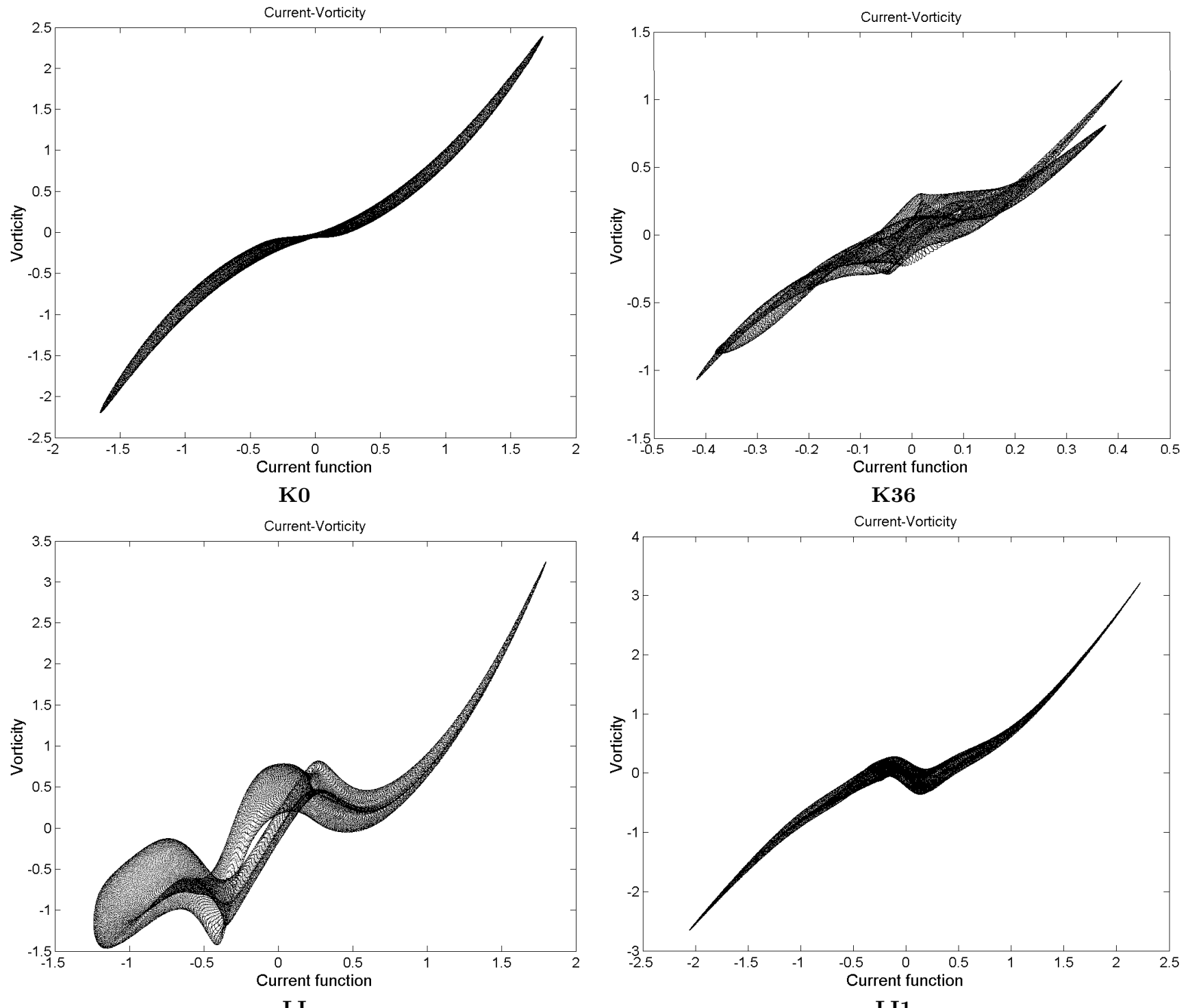

LI

LI1

FIG. 5: Functional relation between the vorticity and the stream function for various simulations (K0, K36, LI and LI1).

short enough to neglect viscous effects and (rare) large fluctuations leading to strong interaction with other domains. These characteristics length and time are related to the size and lifetime of coherent structures, respectively. We use a functional integral method to compute the partition function of a system of point vortices. Let the potential vorticity be concentrated on delta functions

$$
\Omega(\boldsymbol{r}, t)=\sum_{i=1}^{N} \Gamma_{i} \delta\left(\boldsymbol{r}-\boldsymbol{r}_{i}(t)\right) .
$$

We consider $N_{+}$vortices with circulations $\Gamma_{i}=\Gamma_{+}(i=$ $\left.1, \ldots, N_{+}\right)$and $N_{-}$vortices with circulations $\Gamma_{i}=\Gamma_{-}$ $\left(i=N_{+}+1, \ldots, N\right)$, moving in a region $\mathcal{D}$ (in general much smaller than the system area $\mathcal{A}$ ). The correspond- ing stream function is

$$
\psi(\boldsymbol{r}, t)=\frac{1}{2 \pi} \sum_{i=1}^{N} \Gamma_{i}\left(\mathrm{~K}_{0} \kappa\left|\boldsymbol{r}-\boldsymbol{r}_{i}(t)\right|\right)
$$

where $\kappa=1 / l$, and $\mathrm{K}_{0}$ is the modified Bessel function of order zero; we neglect boundary terms, irrelevant in the thermodynamic limit. The Green function of the operator $-\Delta+\kappa^{2}$ at the point $\boldsymbol{r}_{0}$ reduces to the simple unbounded domain expression: $G_{\kappa}=(2 \pi)^{-1} K_{0}(\kappa \mid \boldsymbol{r}-$ $\left.\boldsymbol{r}_{0} \mid\right)$. The position of vortex $i, \boldsymbol{r}_{i}$, satisfies the equation of motion

$$
\dot{\boldsymbol{r}}_{i}=\left.\nabla \psi\right|_{\boldsymbol{r}_{i}} \times \hat{z}
$$

the overdot meaning time derivative. (A rigorous derivation of the motion equations of point vortices, using the 
distribution formalism, may be found in Marchioro \& Pulvirenti [25].) These point vortices were first introduced by Stewart [42], in his study of vortical atmospheric motion in the geostrophic approximation.

The interaction of two geostrophic vortices is similar to the Euler vortices for short distances, the Bessel function tends to $\mathrm{K}_{0}(r / l) \rightarrow-\log r$ for $r \ll l$, but at large distances a screening effect appears, the interaction becomes exponentially weak $\mathrm{K}_{0}(r / l) \rightarrow(\pi l / 2 r)^{1 / 2} \exp (-r / l)$, for $r \gg l$. In addition, we suppose the domain area finite to ensure the convergence of the integral that defines the partition function (see (16)). In fact, at variance to the Onsager remark that in the case of Euler vortices, negative temperature states appear in the infinite area (two-dimensional volume) limit, in the geostrophic case, this hypothesis might not be essential to obtain negative temperature states. Indeed, we will see that one of the consequence of the screening effect of the point vortex interaction at large distances is that localized vorticity equilibrium states, corresponding to negative temperature states, exist, confirming this assumption.

Therefore we postulate that the subsystem of point vortices can be described by a canonical distribution, instead of the microcanonical distribution, that is appropriate for an isolated system. The partition function writes,

$$
Z(N, \beta)=\mathcal{D}^{-N} \int \prod_{i=1}^{N} d \boldsymbol{r}_{i} \exp (-\beta H),
$$

where $\beta$ is the inverse temperature and can take arbitrary real values, and $H$ the Hamiltonian,

$$
H=\sum_{i \neq j} \Gamma_{i} \Gamma_{j} G_{\kappa}\left(\boldsymbol{r}_{i}, \boldsymbol{r}_{j}\right)
$$

We consider the Hamiltonian as being the only relevant integral of motion. It can easily be generalized to take into account the angular momentum conservation. In our case it is not necessary because we consider systems, and also any particular subsystem, without global rotation (the mean vorticity vanishes).

The computation of the partition function is greatly simplified using, instead of the nonlocal Hamiltonian, the local action functional

$$
S_{N}[z]=\frac{1}{2 \beta} \int d \boldsymbol{r} z(\boldsymbol{r})\left(\kappa^{2}-\Delta\right) z(\boldsymbol{r})-i \int d \boldsymbol{r} z(\boldsymbol{r}) \Omega(\boldsymbol{r}),
$$

and the identity

$$
\exp (-\beta H)=\int D z \exp \left\{-S_{N}[z]\right\},
$$

where the dependence on $N$ of the action is through the vorticity $(13), z(\boldsymbol{r})$ is an auxiliary field introduced by the transformation (19), and $D z$ is the functional integral measure. A straightforward computation allows us to relate the field $z$ to the stream function. Indeed, the extremum of the action (18) is given by

$$
\frac{\delta}{\delta z} S_{N}\left[z_{0}\right]=0 \Rightarrow\left(\kappa^{2}-\Delta\right) z_{0}(\boldsymbol{r})=i \beta \Omega(\boldsymbol{r}),
$$

then we can identify the most probable path $z_{0}$ with the stream function, $\psi=-i z_{0}(\boldsymbol{r}) / \beta$. Obviously, using the Gaussian character (in $z$ ) of the integral (19), one can compute it exactly by the Laplace method: replacing back $z_{0}$ in $S_{N}[z]$, one finds again $\exp (-\beta H)$.

The advantage of the representation (19) is that now we can make the integration over the vortex positions $\boldsymbol{r}_{i}$. Indeed, from the expression of $\Omega(13)$, we see that the term in $\Omega$ of the action, has the form $\int d \boldsymbol{r} z \Omega=$ $\sum_{i} \Gamma_{i} z\left(\boldsymbol{r}_{i}\right)$. Therefore, the terms depending on $\boldsymbol{r}_{i}$ in the partition function can be factorized (grouping of terms with the same label $i$ ), and their contribution is finally a factor of the form

$$
\mathcal{D}^{-N} \prod_{ \pm}\left[\int d \boldsymbol{r} e^{i \Gamma_{ \pm} z(\boldsymbol{r})}\right]^{N_{ \pm}}
$$

We are interested in the limit of large $N$. The energy of a system of point vortices grows as $N^{2}$ as demonstrated by Pointin and Lundgren [36], suggesting the scaling $z \rightarrow N z$. To keep the circulation and the stream function finite, we must simultaneously change the temperature scale $\beta \rightarrow \beta N$, and the circulation scale $\Gamma_{ \pm} \rightarrow \pm \Gamma / N$; the total circulation becomes then $\Gamma\left(n_{+}-n_{-}\right)$, with $n_{ \pm}=N_{ \pm} / N$. Remember that $\beta$ is normalized to the area of the system, so that $\beta N$ remains finite in the thermodynamic limit if $N / \mathcal{D}=$ const. Using these scalings the action transforms as $S_{N}[z] \rightarrow N S[z]$, and eliminating the irrelevant multiplicative constants, we obtain the expression

$$
Z(N, \beta)=\int D z \exp \{-N S[z]\},
$$

with

$$
\begin{aligned}
S[z]= & \frac{1}{2 \beta} \int d \boldsymbol{r} z(\boldsymbol{r})\left(\kappa^{2}-\Delta\right) z(\boldsymbol{r})- \\
& \sum_{ \pm} n_{ \pm} \log \int \frac{d \boldsymbol{r}}{\mathcal{D}} e^{\Gamma_{ \pm} z(\boldsymbol{r})}
\end{aligned}
$$

Note that the $O(1 / N)$ boundary terms, do not contribute to the action $S$ in the large $N$ limit, and have been omitted in (22). The functional integral (21) can be computed by the Laplace method in the limit $N \rightarrow \infty$ to find the free energy $f$,

$$
-\beta f\left(\beta, n_{ \pm}\right)=\lim _{N \rightarrow \infty} \frac{1}{N} \log Z(N, \beta) .
$$

The extremum of the action, corresponding to the most probable state, gives the desired mean field equation to the vorticity distribution,

$$
\begin{aligned}
\left(\kappa^{2}-\Delta\right) \psi(\boldsymbol{r})= & \frac{\Gamma n_{+}}{\int d \boldsymbol{r} e^{-\Gamma \beta \psi(\boldsymbol{r})}} e^{-\Gamma \beta \psi(\boldsymbol{r})}- \\
& \frac{\Gamma n_{-}}{\int d \boldsymbol{r} e^{\Gamma \beta \psi(\boldsymbol{r})}} e^{\Gamma \beta \psi(\boldsymbol{r})} .
\end{aligned}
$$

this is equivalent to the equation found by Joyce \& Montgomery (1973), with the extra term in $\kappa$. Solutions of 
(24) make the free energy (23) extremal, then giving a thermodynamically favorable state.

Of particular interest are the localized solutions, if they exist, because their superposition would evolve only slowly in time. Indeed, if $\psi_{1}$ and $\psi_{2}$ are two solutions of (24), then, introducing $\psi=\psi_{1}+\psi_{2}$ into (2), we see that the time derivative of the total vorticity is of the order of the overlap of the two solutions. For two exponentially localized solutions this overlap is exponentially small. This means that if some region of the flow approaches a solution of the thermodynamic vorticity equation, the resulting structure would not show significant variations before a long time. We show in the next section that what is observed in the simulations, is consistent with this picture.

\section{ASYMPTOTIC METHOD AND LOCALIZED VORTICITY SOLUTIONS}

The question which naturally arises is whether the coherent structures observed numerically correspond to local thermodynamic states, solution of (24). Are the relations vorticity-stream function of figure 5 representative of solutions of the thermodynamic state equations? The comparison of the vortices observed numerically and explicit solutions of (24), with the relevant physical parameters, is the main goal of the present section.

\section{A. The thermodynamic equation}

There is a deep difference in the mathematical structure of the Euler and geostrophic mean field equations. Although for positive temperatures $(\beta>0), \Delta \psi=$ $-\omega(\psi)$ is a monotone increasing function, and the usual theorems of existence and uniqueness of nonlinear elliptic equations apply [43], for negative temperatures the behavior of both systems differs. In the Euler case, the equilibrium equation turns out to be completely integrable, and can be solved, by analogy with the sineGordon equation, using the inverse scattering transform [44]. In the geostrophic case the term in $\kappa$ makes $\omega(\psi)$ non-monotonic, as we discussed in the introduction, and then the free energy may have more than one minimum, so the existence and uniqueness of the solution are not guaranteed.

A rich variety of solutions of the Euler flow appears when additional constraints or more complicated boundary conditions are considered. Complicated bifurcations of the set of solutions of the thermodynamic equations are found for instance in a bounded domain as shown by Chavanis and Somméria [7], or in a subdomain of an unbounded field when the effects of circulation, impulse, and angular momentum conservation are taken into account [9]. However, in these cases for a given set of parameters and boundary conditions, the solution of the thermodynamic equation is unique. At variance, in the geostrophic case, the fact that the $\omega(\psi)$ relation is nonmonotonic opens the possibility of having simultaneously different solutions.

In this section we introduce an iterative asymptotic method which allows us to find explicit (particular) solutions of (24). These solutions in the form of isolated vortices are therefore representative of local thermodynamical equilibrium states, and will be compared with the vortices appearing in the numerical simulations.

The thermodynamic state determined by the solution of equation (24), rewritten in a form similar to (10),

$$
\Delta \psi=-\omega(\psi)=\kappa^{2} \psi+\alpha_{-} \mathrm{e}^{\beta \Gamma \psi}-\alpha_{+} \mathrm{e}^{-\beta \Gamma \psi},
$$

depends on various parameters, in particular $\beta$, the inverse of an energy, and $\Gamma$ a circulation. The combination $\beta \Gamma$ appears as the inverse of a characteristic amplitude of the stream function (the coefficient in the exponentials of equation (25)). For a given initial condition, the system evolves in such a way that a specific $\omega-\psi$ relation establishes, as shown in figure 5 , and then a specific value of $\beta \Gamma$. In fact, after a rescaling transformation of (25), using $1 / \beta \Gamma$ as the unit of the stream function, the number of parameters is reduced, meaning that for a given solution of the scaled thermodynamic equation, a whole family of states are obtained, that may be compared to the numerical results.

It is therefore convenient to rewrite (24) in terms of the new scaled (nondimensional) variable $\beta \Gamma \psi \rightarrow \psi$. This change is equivalent to a change in the time units of the original dynamical equations (Hamiltonian of point vortices or Euler). Indeed, the original equations are invariant under the scaling transformation $(\psi \rightarrow \beta \Gamma \psi, t \rightarrow$ $t /(\beta \Gamma))$, for arbitrary $\beta$. We hence have,

$$
\Delta \psi=g(\psi), \quad g(\psi)=\kappa^{2} \psi+\alpha_{-} \mathrm{e}^{\psi}-\alpha_{+} \mathrm{e}^{-\psi},
$$

where $g$ is the scaled vorticity, and $\alpha_{ \pm}$are now

$$
\alpha_{ \pm}=\beta \Gamma^{2} n_{ \pm}\left[\int d \boldsymbol{r} \mathrm{e}^{\mp \psi}\right]^{-1} .
$$

We verify that in the new form, $\beta$ and $\Gamma$ only appear through the combination $\beta \Gamma^{2}$, the number of parameters was then reduced by one. The integral of the two last terms of $g(\psi)$ is proportional to the mean potential vorticity, and satisfies,

$$
\int d \boldsymbol{r} \Omega=\int d \boldsymbol{r}\left(\alpha_{+} \mathrm{e}^{-\psi}-\alpha_{-} \mathrm{e}^{\psi}\right)=\beta \Gamma^{2}\left(n_{+}-n_{-}\right) .
$$

Solutions of (26) may depend on the independent nondimensional parameters $\left\{\kappa, n_{+}, \beta \Gamma^{2}\right\}$ (or $n_{-}$instead of $n_{+}$ since they are related by $\left.n_{-}+n_{+}=1\right)$. Moreover, from the definition (27) of $\alpha_{ \pm}$we also have the integral relation

$$
\int d \boldsymbol{r}\left(\alpha_{+} \mathrm{e}^{-\psi}+\alpha_{-} \mathrm{e}^{\psi}\right)=\beta \Gamma^{2} .
$$

These two relations (28) and (29), can be interpreted as a set of compatibility conditions relating the family of parameters $\left\{\alpha_{-}, \alpha_{+}\right\}$to the set $\left\{n_{+}, \beta \Gamma^{2}\right\}$. 


\section{B. Vortex in a local thermodynamic state}

The strong, isolated, positive vortex observed in the long term evolution of the LI simulation $(t=600)$, is a good candidate to represent a local equilibrium state. It has a well defined vorticity-stream function relation, at least in the $\psi \gtrsim 0$ range (see figure 6 ), relation guarantying that it is in a relative stationary state. Its vorticity distribution is, in addition, almost axisymmetric (c.f. figure 7 below), facilitating analytic calculation because it should be enough to assume $\psi(x, y)=\psi(r)$, with $r$ a polar radial coordinate, and origin placed at the vorticity maximum. We start then by fitting the scattered points of the $\omega(\psi)$ relation of figure 5 (LI simulation). The fit, a superposition of exponentials, corresponds to the function $\omega(\psi)$ of Eq. (25), is shown in figure 6(a).

The observed relation is distorted in the $\psi \lesssim 0$ region by small amplitude vorticity structures surrounding the main vortex. To minimize the effect of this noise, we essentially take points in the $\psi \gtrsim 0$ range. In addition, the dispersion of the observed points in the $\omega(\psi)$ graph, even in the "main vortex" region, is obviously due to the influence of both nonstationary and viscous effects. This dispersion introduces some error in the value of the parameters of the fit, nevertheless, in practice it turns out that several digits are necessary to keep the fitting curve within the point cloud. We obtain $\omega(\psi)=36 \psi+59.063 \exp (-0.301 \psi)-59.113 \exp (0.301 \psi)$, and after the rescaling $\beta \Gamma \psi \rightarrow \psi$ with $\beta \Gamma=-0.301$, we find $g(\psi)$ with the parameters $\alpha_{-}=-17.778$ and $\alpha_{+}=-17.793$, as shown in figure 6(b). It is worth noting that the functional relation just obtained, which is a consequence of the thermodynamic approach, is consistent with the observed vorticity-stream function relation.

The fit $g(\psi)$ is not enough to ensure that the actual vorticity distribution satisfy Eq. 26, we must also verify if the solution obtained from this functional relation has physically admissible parameters (such as $n_{ \pm}$), and the vortex shape agrees with the observed one. Indeed, consistence of the vorticity-stream function relation of a stationary coherent structure with the thermodynamic theory does not mean that the structure is related to the equilibrium solution of the thermodynamic equation: this last equation might not give a solution comparable to the observed vortex even for a fixed form $g$.

\section{Piecewise linear approximation}

Before computing an explicit solution $\psi$ of (24), it is enlightening and useful to search a piecewise linear approximation $\psi_{L}$ of the problem, i.e. a solution of the equation

$$
\Delta \psi_{L}=g_{L}\left(\psi_{L}\right)
$$

where the function $g_{L}$ is a piecewise linear approximation of the scaled vorticity $g$. Details about the construction of $\psi_{L}(r)$ can be found in appendix B. We show in figure 6 (dashed line) a $g_{L}$ made up of $M=3$ line segments. The index $L$ emphasizes that the quantity is evaluated using the piecewise linear vorticity. In a first step we solve the approximated version of the thermodynamic equation, and then, we will use this solution as an initial guess to numerically compute the smooth vorticity satisfying (24).

Let us note that within this approximation, the problem is reduced to a set of solvable linear equations and matching conditions. However, a difficulty remains, related to the fact that the coefficients $\alpha_{ \pm}$are functionals of the solution $\psi$. They are linked to some integral properties of the flow such as, for instance, the mean potential vorticity (28). To take into account these properties it will appear necessary, also, to appropriately approximate these functionals (cf Appendix B). Because of this set of compatibility constraints, we finally deal with a simplified but still nonlinear algebraic problem.

The boundary conditions are specified at the radius $R$ of the solution, implicitly defined by $\psi_{L}(R)=\psi_{1}=0.004$ and $\psi_{L}(0)=\psi_{M}=-0.542$, the maximum value. The key point, determining the shape of the solution, is that the sign of the slope in the edge region differs of the one in the core. Indeed in each interval the solution of (30) can be expressed as a superposition of (zero-order) Bessel functions, the general solution is

$$
\begin{gathered}
\psi_{L}(r)=A_{3} \mathrm{~J}_{0}\left(\kappa_{3} r\right)+C_{3}, \psi \in I_{3}, 0 \leqslant r<R_{23} \\
\psi_{L}(r)=A_{2} \mathrm{~J}_{0}\left(\kappa_{2} r\right)+B_{2} \mathrm{Y}_{0}\left(\kappa_{2} r\right)+C_{2} \\
\psi \in I_{2}, R_{23}<r<R_{12} \\
\psi_{L}(r)=A_{1} \mathrm{~K}_{0}\left(\kappa_{1} r\right)+B_{1} \mathrm{I}_{0}\left(\kappa_{1} r\right)+C_{1} \\
\psi \in I_{1}, R_{12}<r<R
\end{gathered}
$$

where $\left\{A_{i}, B_{i}\right\}$ and $C_{i}$ (with $i=1,2,3$ ) are constants fixed by the shape of $g_{L} ; \kappa_{i}$ are the square root of the absolute values of the three $g_{L}$ slopes; $R_{23}=1.066$ and $R_{12}=1.797$ are the radial values where the linear solutions $\psi_{L}(r) \bigcap I_{i}$ are matched. The linear approximation of the radius is $R_{L}=2.375$ (for details on matching conditions, cf Appendix C, available online [49]).

The solution $\Delta \psi_{L}(r)$ of (30) is drawn in figure 7 in the unscaled variables (dashed line). It is found that $\psi_{L}$ is a monotone increasing function with a single minimum (a maximum of $-\psi_{L}$ ) located at the origin, as observed in the numerical simulation. Monotony of the solution is not a trivial result: non physical solutions with multiple maxima can be found by modifying the slopes of $g_{L}$. The first term in (33) contains the modified Bessel function $\mathrm{K}_{0}$, which decays exponentially for large $r$. The behavior of the vorticity tail will obviously depend on the actual values of the coefficients $A_{1}$ and $B_{1}$. However, in this interval $\psi$ is small and the coefficient of the exponentially increasing $\mathrm{I}_{0}\left(\kappa_{1} r\right)$ Bessel function, must in fact be a rapidly decreasing function of the disc size $R_{L}$ (if we relax the (pseudo-)boundary constraint $\psi_{L}(0)=\psi_{M}$ and fix the radius $R_{L}$ ), which should ensure the exponential decay of $\psi_{L}$ or $\psi$. 


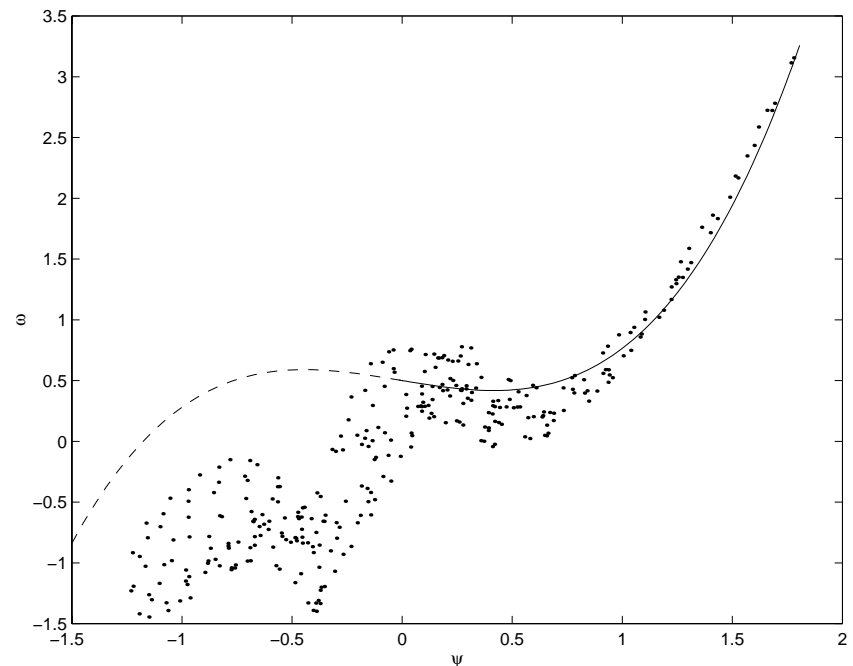

(a)

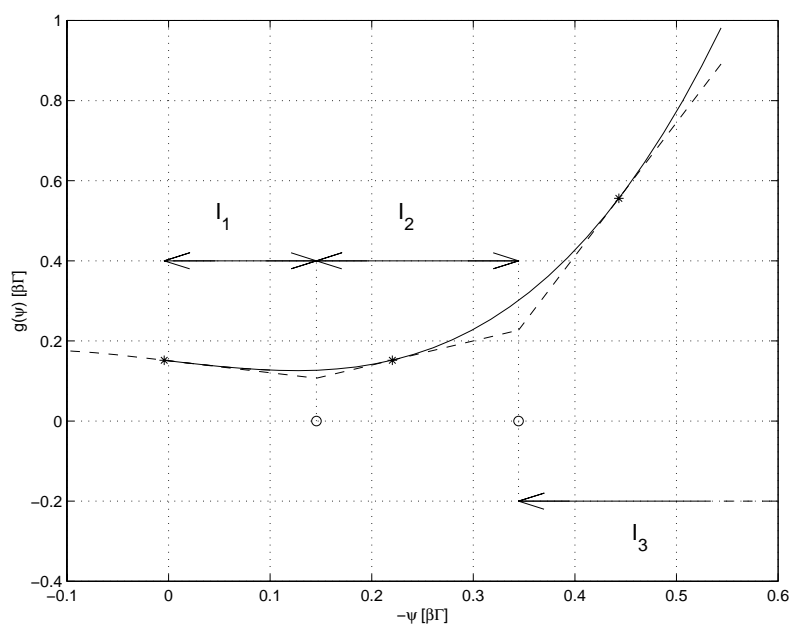

(b)

FIG. 6: Fit of $\omega(\psi)$ (from figure 5, LI) using the function (25). (a) $\alpha_{-}=59.063, \alpha_{+}=59.113$, and $\beta \Gamma=-0.301$ (solid and dashed line); (b) normalized vorticity $g(\psi)$, in units of $\beta \Gamma$, and its piecewise linear approximation $g_{L}(\psi)$ (dashed line), in the adjacent intervals $I_{i}(i=1,2,3)$, around the tangent points $\psi_{i}$ (stars) and separated by the matching points $\psi_{12}$, and $\psi_{23}$ (circles).

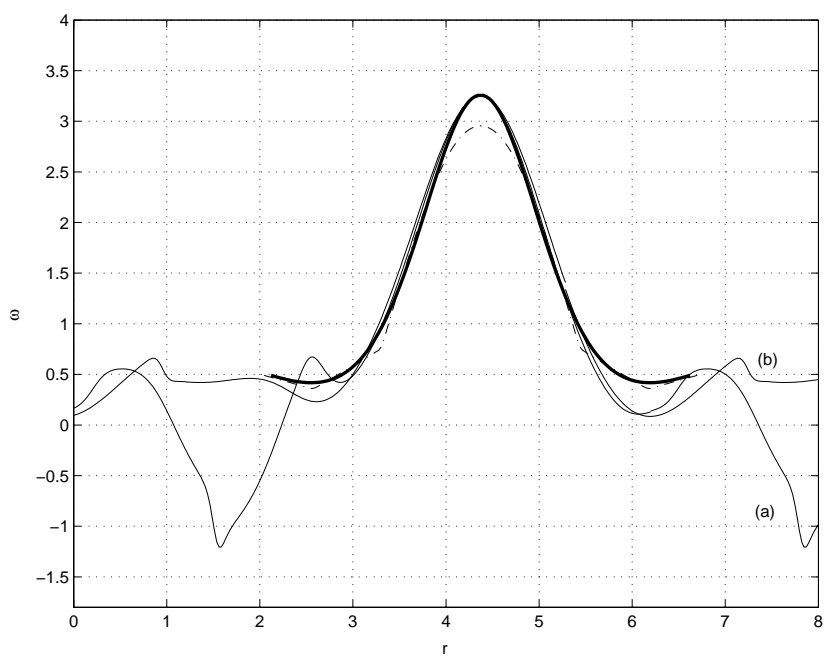

FIG. 7: Comparison of the vortex observed in the LI simulation at $t=600$ (c.f. figure 4 ) and the vortex computed using the thermodynamic equation. Solid lines: two orthogonal profiles of the observed vortex (labels (a) and (b)). Dot-dashed line: piecewise approximation of the equilibrium vorticity (in the unscaled variables). Thick solid line: exact thermodynamic equilibrium vorticity.

Now, using the compatibility constraints we can compute the fraction of positive vortices $n_{+}=0.602$ and the temperature parameter $\beta \Gamma^{2}=-651$. Recalling that we fixed the free parameter $\beta \Gamma$ equal to -0.301 , we can deduce $\beta=(\beta \Gamma)^{2} /\left(\beta \Gamma^{2}\right) \sim-1.410^{-4}$. We see below that the exact (numerical) solution give similar values, qualitatively validating the piecewise approximation.

\section{Numerical exact solution of the thermodynamic equation}

To get the exact solution $\psi(r)$, we start from $\psi_{L}$ and proceed by recurrence (the convergence of the method is proven in the online appendix D [49]). More precisely, we construct an analytic continuation of the computed stream function $\psi_{L}, \psi_{L}^{a c}$ : the initial guess is $\psi_{0}=\psi_{L}^{a c}$ from which the radius is $R_{0}=R_{L}$. Analytic continuation is necessary to get an asymptotic solution satisfying the boundary conditions. Indeed $R=R_{\infty}$, the radius of $\psi$, is a function of the boundary conditions and might be greater than the initial radius $R_{L}$.

The iterative procedure follows as: starting with the know value of $\psi_{n},[\Delta \psi]_{n+1}$ is computed using $[\Delta \psi]_{n+1}=$ $g\left(\psi_{n}\right)$; then $\psi_{n+1}$ is deduced by inversion of the laplacian (i.e $\left.\Delta \psi_{n+1}=[\Delta \psi]_{n+1}\right)$. At each step three constraints must be satisfied, (a) $\psi_{n}(0)=\psi_{M}$, (b) $\psi_{n}(0)$ is a minimum, and (c) $\Delta \psi_{n}(0)=-\omega_{M}$; the value obtained in the simulation is $\omega_{M}=-0.98$ (see the figure 6). As can be shown, these constraints being true for $n=0$ by construction, remain true for any $n$. The radius of the solution $R=R(n)$ is by definition such that the vorticity $\omega_{n}(R)=0.15(\sim 0.5$ in unscaled units, see figure 6$)$. In figure (8), we show the evolution of the physical parameters, i.e $\beta(n), n_{+}(n), \Gamma(n)$ and $R(n)$, as a function of the number of iterations $n$. We note that, owing to the chosen initial condition, convergence of the scheme is very fast and gives $\beta=-1.0510^{-3}, n_{+}=0.652, \Gamma=288$, and $R=2.25$ (for $n \rightarrow \infty$ ).

As expected, the radius of the solution $\omega(r)$ is similar to the one we get by the piecewise linear approximation, as shown in figure 7 . In fact, it turns out that $\omega(r) \sim g\left(\psi_{L}(r) / \beta \Gamma\right) / \beta \Gamma$ : the method of inserting the so- 

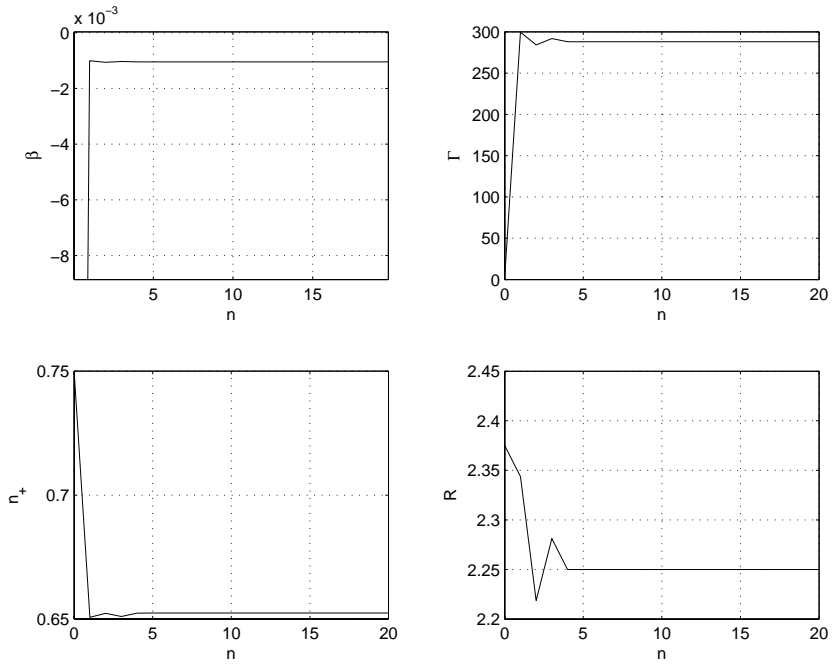

FIG. 8: Evolution of the parameters $\beta, n_{+}, \Gamma$ and $R$ as a function of the number of iterations $n$ showing a rapid convergence of the scheme.

lution $\psi_{L}$ obtained using $g_{L}$ into $g$ can be interpreted as an accelerated convergence procedure equivalent to take the limit $M \rightarrow \infty$ (see also Appendix D online [49]). We observe a posteriori that $M=3$ has given qualitative accurate results in reproducing the exact solution of the thermodynamic equation. The agreement between the the two methods is rather good for the fraction of positive vortices $(0.602$ and 0.652$)$. There is however a significant difference between the values obtained for the $\beta \Gamma^{2}$ parameter. Two reasons may explain such a difference, first the sensitivity of the nonlinear algebraic problem in the shape of $g_{L}$ (see Eqs. (B2)-(B4)), and then the kind of parametric dependency which is exponential for $\beta \Gamma^{2}$ and only linear for $n_{ \pm}$.

\section{Discussion}

We first note that as expected, the temperature is negative (positive temperature states are homogenous), and that, in agreement with its physical definition, the positive vortex density $n_{+}$is in the range $[0,1]$. From a mathematical point of view, the definition of $n_{+}$does not restrict its admissible values to the interval $[0,1]$. $A$ priori, only a limited set of values of parameters $\left(\alpha_{-}, \alpha_{+}\right)$ is compatible with this constraint. In other words, there was a selection of the parameters $\left(\alpha_{-}, \alpha_{+}\right)$by the dynamics, which resulted to be compatible with the hypothesis made to obtain the thermodynamics equations. This is a striking confirmation of the relevant character of the thermodynamical approach with only two species of point vortices (with circulation values $\pm \Gamma$ ). Indeed, one could think that to reproduce the results of the simulations, one would have to take an infinity number of vortex "species", having different circulations. In fact, the thermodynamic equation (24) deduced with a distribution of positive and negative vortices of equal circulation, turn out to be sufficient to reproduce the observed state.

Another hypothesis used in this construction was that, for the given set of the parameters $\left\{\alpha_{-}, \alpha_{+}\right\}$, the stream function should be strictly decreasing (with $r$ ), in order to match the observed vortex. This is not necessarily the case for arbitrary values of these parameters, and it may result in a vortex possessing, for instance, more than one maximum, or even, in the absence of solution at all. However, for the parameters found in the simulation, this property could be respected, and the shapes of the observed and computed vortices, a posteriori agreed. This is an important point which also means that the long time evolution of the system is consistent with the formation of localized thermodynamic states, in the form of coherent structures.

In figure 7, we display two orthogonal cuts of the vortex profile observed in the LI simulation (the solid lines labelled (a) and (b)). These cuts pass through the vorticity maximum, which should be the axis of symmetry of the vortex. We indeed see, on the one hand, that the main vortex is almost axially symmetric and, on the other hand, that some other smaller vortex structures are present only in the boundary region of the vortex. We also superposed on the same figure, the calculated solutions (dashed line and thick solid line) obtained using the thermodynamic theory. To solve this equation we used an approximated method based in a piecewise fit. We obtained a solution having a maximum significantly lower than the actual one, in part because the condition at $r=0$ was applied to the stream function $\psi(0)=\psi_{M}$ and not directly to the vorticity. The numerical method consisted in an iterative process based on a step by step refinement of the piecewise linear approximation. This method turns out to be rapidly convergent and gives a vortex very close to the observed one.

It is worth noting that the value of the parameters defining the shape of the vortex, like for instance $R$ and $\psi_{M}$ in the piecewise approximation, are strongly dependent. This implies that the shape of the vortex is extremely sensitive to variations in their values. For example, if instead of $R \approx 2.4$ we used $R=2.2$, the maximum of the vorticity would attain a value around 5 instead of 3. Therefore, the error in the computed profile is rather small (the allowed range of consistent parameters is small).

The size of the region in local equilibrium must obviously be much larger than the interaction length $l$. The solution we obtained occupies a region of characteristic size $R$, which can be interpreted as the "box" length $l_{B}$ we mentioned in the Introduction. We verify that $l \ll R \sim l_{B} \lesssim L$. In general, the simulations show that the system evolves to a state with several well differentiated regions in local equilibrium; in K36 four of such regions coexist, in LI1 two well separated positive and negative vortices are present. Each of these regions have their own set of thermodynamic parameters. The processes of merging of like sign vortices, which have cre- 
ated these regions, appears to be faster than the time to get a global equilibrium. The obtained value of the positive circulation fraction $n_{+}$, larger than $1 / 2\left(n_{+}>n_{-}\right)$, is a reflect of this situation. Global equilibrium requires equality $n_{+}=n_{-}$.

In this approach we neglected some effects which can modify the vorticity-stream function relation as for example the possible departures from the Boltzmann distribution and the viscosity. Indeed, we observed in the simulations that the vorticity tend to vary slower than exponentially at large amplitudes (see also the simulations presented by Matthaeus et al. [27], and Segre \& Kida [39]). The thermodynamic theory based on a continuous distribution of vorticity, and taking into account the whole family of invariants, of Robert \& Somméria [37], and Miller et al. [30], gives a Fermi distribution (which in the special 2 -species case implies $\Omega \sim \tanh (\psi)$ ) rather than a Boltzmann distribution as given by the present point vortex theory $(\Omega \sim \sinh (\psi))$. The Boltzmann approximation is valid in the limit $|\beta \Gamma \psi| \ll 1$ and in our case the maximum value of $|\beta \Gamma \psi|$ is about 0.5 . In other words, we are near the limit where the Boltzmann approximation is valid (dilute gas or dilute vorticity approximation). Besides, the damping effect due to the viscosity, which is stronger on the vorticity than on the stream function, may also explain this flatness of the observed vorticity-stream function relation.

In summary, the vortex obtained using the thermodynamic equation is in agreement with the vortex observed in the simulation, which confirms that the flow evolves to states (coherent structures) locally in thermodynamical equilibrium.

\section{CONCLUSIONS}

In this paper we studied the motion of a rotating fluid layer in two-dimensional decaying turbulence, with characteristic temporal variations much smaller than the rotation frequency. The resulting quasi-geostrophic flow is mathematically analogous to the ion drift motion in a magnetized plasma. We studied the phenomenology of this system by direct numerical simulation, using a pseudo-spectral code. To avoid the concentration of energy at small scales, which may induce numerical instabilities, a viscous term has been added in the simulation.

It has been shown, that in this regime of decaying of an initial random state, the Rossby length $l$ play an essential role in the relaxation process towards equilibrium states. Indeed, at variance to the Euler dynamics which corresponds to the special case $l=+\infty$, no global quasistationary state is reached despite the very long simulation time, of hundreds of eddy turnover periods (and up to times of the same order as the typical viscous time), when $l$ is small compared with the characteristic length of the box. We observed instead that for finite $l$, the system evolves towards spatially localized quasi-stationary states. The typical life times of these states is much larger than their turnover time, and may reach the order of the viscous time. A similar scaling of the flow complexity with its size was also noted for the Hasegawa-Mima system [12].

The observed long lived vortices, which appear after a first rapid evolving stage dominated by vortex merging, are well separated coherent structures, generally surrounded by small amplitude vorticity fluctuations. The coherent structures evolve inside regions of non-vanishing mean vorticity, separated by strong gradients having sometimes a scale length comparable to the interaction length. One important property of these coherent structures, is that they obey a vorticity-stream function relation (this may be taken as the definition of the coherent structure [12]). Therefore, the question about the nature of these states and their relation to some thermodynamic equilibrium state arises naturally.

In order to characterize these quasi-equilibrium states, we have developed a thermodynamical theory, using a point vortex approach. The goal of this theory is to obtain the most probable vorticity distribution, in the sense of the entropy. The difference with respect to the usual theories of thermodynamic hydrodynamics, is that we apply this approach not to the whole system, but only to the regions staying in a quasi-stationary state. This approach allows an easier justification of the different approximations needed to obtain the mean thermodynamic vorticity equation. One condition for the validity of the thermodynamical approach is that the system of point vortices be "mixing" [25], [45]. In fact, as our goal was to apply thermodynamics to a subsystem, that is in some localized region and for a limited period of time, the assumption of mixing, and even of the existence of the thermodynamic limit is less restrictive than in the general case of the global relaxation of Euler flows because of the presence of energy fluctuations. Moreover, our approach using point vortices, instead of continuous distribution of vorticity, is coherent with the local equilibrium assumption: firstly, an arbitrary distribution of vorticity can be fitted by a set of point vortices at a given time; secondly, the error in the fit does not grow in time, if the number of point vortices tends to infinity, this is precisely the thermodynamic limit.

We used the canonical ensemble and worked with a local functional action instead of the nonlocal point vortex Hamiltonian. We obtained a mean field equation for the most probable vorticity distribution by minimizing the free energy functional. We showed that this equation possesses not only global equilibrium solutions (as in the Euler case), but can also describe localized structures, in agreement to our goal of describing local thermodynamic states.

The thermodynamic equation is a non-integrable and nonlinear integro-differential equation. To solve this equation we proceeded first with an approximated method using a piecewise linear fit of the vorticity-stream function relation; then we used this method as the base of an iteration algorithm to solve the equation numer- 
ically. The asymptotic method allowed us to compute explicit solutions and to reproduce the main properties of the vorticity distribution.

From the numerically exact solution we checked that, up to typical fluctuations around the boundary of the vortex, there is a very good correspondence between the thermodynamic state, solution of the mean field equation, and the observed vortex in the numerical simulations (having the same parameters). The observed and computed vortices are similar, with comparable amplitudes and occupying regions having the same size. The temperature of this state is negative, and the proportion of positive circulation is greater than the negative one. This last property emphasize the local character of the state as its thermodynamic parameters are different from the global ones (the mean vorticity is of course zero). This clearly shows, and this is one of the main conclusions of the present work, that the coherent structures naturally formed during the system's long term evolution, are in fact local thermodynamical equilibrium states. It would be interesting to test these theoretical results by experiment, using for instance, a device similar to the one used by the Driscoll group [13]. In order to obtain the effects related to the potential vorticity, the particles in the device should have a non negligible Larmor radius.

\section{Acknowledgments}

Thanks to P.-H. Chavanis, S. Le Dizès, Y. Pomeau and J. Somméria for useful comments and discussions. This work has been supported by the Centre National de la Recherche Scientifique, and Universités d'Aix-Marseille I \& II (UMR 6594).

\section{APPENDIX A: NUMERICAL METHOD}

The spatial discretisation is made using fast Fourier transforms. Nonlinear terms are first computed in real space, at each time step, and after transformed back to Fourier space. We use the 2/3-rule for antialiasing. Each Fourier mode of the stream function, say $a(t)$, satisfies an equation of the form

$$
\dot{a}(t)=L a(t)+N(a(t)),
$$

where $L$ is the linear term and $N(a)$ the nonlinear one. One time step $a(t+\Delta t)$ consists in a predictor step $\tilde{a}=$ $\tilde{a}(t+\Delta t)$

$$
\tilde{a}=a(t-\Delta t)+\left(e^{L \Delta t}-e^{-L \Delta t}\right) a(t)+2 \Delta t N(a(t)),
$$

which is a modified leap-frog, and in a corrector step,

$$
\begin{aligned}
a(t+\Delta t)= & a(t)+\frac{1}{2}\left(e^{L \Delta t / 2}-e^{-L \Delta t / 2}\right)(a(t)+\tilde{a})+ \\
& \frac{\Delta t}{2}[N(a(t))+N(\tilde{a})]
\end{aligned}
$$

The algorithm is globally second order in time, and the nonlinear term is computed twice per step. The exponentials in the predictor step ensure exact integration in absence of nonlinearity. The corrector step is centred around $\Delta t / 2$. This algorithm is a modified version of one of the Gazdag (1976) time-differencing schemes.

\section{APPENDIX B: THE PIECEWISE LINEAR APPROXIMATION}

In order to obtain explicit approximate solutions of the system (26-29), describing the distribution of vorticity in an equilibrium state, we develop an algorithm based on the substitution of the transcendental function $\omega(\psi)$ by a piecewise linear function. In this way the problem reduces to a set of solvable linear problems, and matching conditions. However, a difficulty remains, related to the fact that coefficients $\alpha_{ \pm}$are functionals of the solution $\psi$ and are linked to some integral properties of the flow such as, for instance, the mean potential vorticity (28). To take into account these properties it will appear necessary, also, to appropriately model and approximate these functionals. Because of this set of compatibility constraints, we will finally deal with a simplified but still nonlinear algebraic problem.

We first construct the function $g_{L}(\psi)$, a piecewise approximation of $g(\psi)$ (see below figure 6 , for an example). For any set of intervals $\left\{I_{i}\right\}_{i=1}^{M}$, disjoints and covering the $\psi$ axis, and any set of numbers $\left\{\psi_{i}\right\}_{i=1}^{M}$ with $\psi_{i} \in I_{i}$, we define

$$
g_{L}(\psi)=g^{\prime}\left(\psi_{i}\right)\left(\psi-\psi_{i}\right)+g\left(\psi_{i}\right), \psi \in I_{i}, i=1, \ldots, M .
$$

$M$ is the number of intervals where $g_{L}$ is linear and $g^{\prime}\left(\psi_{i}\right)$ is the derivative of $g$ with respect to $\psi$ at the point $\psi_{i}$. In that way, we clearly satisfy that $g$ and $g_{L}$ are tangent at any point $\psi_{i}$ and, in the limit $M$ tending to infinity and length $\left(I_{i}\right)$ tending to zero for every $i, g_{L}$ approaches $g$. Of course in a specific case a finite value of $M$ is enough to obtain a satisfactory approximate solution of (24).

However, we immediately see that condition (28) cannot be satisfied by this form of $g$ without changing also the integral constraints. Indeed, once the parameters $\alpha_{ \pm}$ chosen, and found the solution of (26) with $g$ replaced by $g_{L}$, one may compute the left and right hand sides of (28) separately. Clearly, by construction they will appear in general to be different. In order to preserve the compatibility conditions (28) and (29), it is necessary to simultaneously change both $g(\psi)$ and the functional form of the parameters $\alpha_{ \pm}$.

The simplest self-consistent piecewise linear formulation of the equilibrium vorticity equation is, in addition to the approximation (B1), to linearize at the same time conditions (28) and (29). To this end, we write

$$
\Delta \psi=\left(A_{-}^{i}+A_{+}^{i}+\kappa^{2}\right)\left(\psi-\psi_{i}\right)+g\left(\psi_{i}\right), \psi \in I_{i},
$$

where the new constants $A_{ \pm}^{i}=\alpha_{ \pm} e^{\mp \psi_{i}}$ satisfy by definition $g\left(\psi_{i}\right)=A_{-}^{i}-A_{+}^{i}+\kappa^{2} \psi_{i}$ (obviously, $g^{\prime}\left(\psi_{i}\right)=$ 
$\left.A_{-}^{i}+A_{+}^{i}+\kappa^{2}\right)$. We also impose

$$
\begin{gathered}
\sum_{i=1}^{M} \int_{J_{i}} d \boldsymbol{r}\left[\left(A_{-}^{i}-A_{+}^{i}\right)+\left(A_{-}^{i}+A_{+}^{i}\right)\left(\psi-\psi_{i}\right)\right]= \\
\beta \Gamma^{2}\left(n_{-}-n_{+}\right),
\end{gathered}
$$

the mean vorticity condition, and

$$
\begin{gathered}
\sum_{i=1}^{M} \int_{J_{i}} d \boldsymbol{r}\left[\left(A_{-}^{i}+A_{+}^{i}\right)+\left(A_{-}^{i}-A_{+}^{i}\right)\left(\psi-\psi_{i}\right)\right]= \\
\beta \Gamma^{2},
\end{gathered}
$$

the "temperature" condition. In (B3) and (B4) the integration domain is over the sets $\left\{\boldsymbol{r} \in J_{i}\right\}$ belonging to the fluid space domain $\mathcal{A}$. The sets $\left\{\boldsymbol{r} \in J_{i}\right\}$ are the image, in $\mathbf{r}$-space, of the intervals $I_{i}$ in $\psi$-space.

The problem reduces then to solve (B2) and (B3-B4) in a given region of the flow, where a well defined relation between vorticity and current function is satisfied, complemented with adequate boundary conditions. If this relation actually represents a state close to a local thermodynamic equilibrium with a specific set of parameters, the solution of (24) having the same parameters, must exist and also have the same shape as the observed vortex. The comparison of the numerical simulations with the states of local thermodynamic equilibrium, solution of (24) consists in the following steps. The first step (i) is to fit the observed $\omega-\psi$ relation (seen in numerical simulations) with the function $\omega(\psi)$ (as defined in (25), this fixes the value of the parameters $\beta \Gamma$ and $\alpha_{ \pm}$, and after scaling of the stream function, the function $g(\psi)$; the second step (ii) consists in defining the piecewise linear function $g_{L}$ (using the nondimensional form), in particular the intervals $I_{i}$ and the points $\psi_{i}$, which determine $A_{ \pm}$; in the third step (iii) one solves the Helmholtz equations in each image $J_{i}$ of $I_{i}$ (which are not known a priori), and the matching and boundary conditions, to obtain the approximated stream function $\psi(\boldsymbol{r})$; next, in the fourth step (iv), using (B3) and (B4)) one determines the global parameters $n_{+}$and $\beta \Gamma^{2}$. If the parameters are physically acceptable (for instance $n_{+} \in[0,1]$, or $\beta<0$ ), and the vortex shape compares well with the observed one, we may conclude that the region analyzed is in a state of local thermodynamic equilibrium.

For fixed $M$ (the number of linear pieces of $g_{L}$ ) this algorithm gives an approximate solution of the full nonlinear problem. The convergence of the algorithm, as the value of $M$ increases (i.e increasing the number of linear intervals), is discussed in Appendix D (online) [49]. Here we use this procedure to obtain a solution from the $o b$ served vorticity-stream function relation (relation which fixes the value of the parameters). Obviously, it also may be used to obtain solutions satisfying some boundary conditions independently of any observed vorticity distribution.

\section{APPENDIX C: EXPLICIT CONSTRUCTION OF THE PIECEWISE LINEAR APPROXIMATED SOLUTION $\psi_{L}$.}

In order to compute this solution, we construct the piecewise linear approximation $g_{L}$ of $g$ (figure $6(\mathrm{~b})$ ). We first define the total interval in $\psi$ to which the vortex belong. (Note that in the new variables $\psi$ is negative as is $\beta \Gamma$, then we use $-\psi$ instead.) The maximum value is easily identified in figure $6(\mathrm{~b})$, it gives $-\psi_{M}=0.542$. We define the minimum value of the stream function interval, the "border" of the vortex, at the inflexion point of the curve $g,-\psi_{1}=-\psi(R)=0.004$. We denoted $R$ the radius of the vortex domain, inside which we solve (B2) and (B3-B4). In fact, $R$ is a parameter which remains to be determined (as the other matching points). We see that the vorticity at $R, g\left(\psi_{1}\right)=\Delta \psi(R)=0.15$, or in the original variables $\omega(R)=0.15 /|\beta \Gamma|=0.5$, is positive, which means that there is a net "background" of vorticity around the vortex. This interval, which starts at the inflexion point, defines a region of definite curvature sign of the function $g$. The sign of the curvature of $g$ is related to the sign of the vorticity. In the simulations, where the total vorticity is zero, as the system evolves the positive and negative vorticity components tend to separate, creating large vortex regions with well defined sign. This justifies the choice of the inflexion point as the boundary of the thermodynamic equilibrium domain.

The first linear piece of $g_{L}$ is defined by the tangent to the curve $g(\psi)$ at $\psi_{1}=-0.004$. Analogously, we define a second point $\psi_{2}$, satisfying $g\left(\psi_{2}\right)=g\left(\psi_{1}\right)=0.15$, and giving $\psi_{2}=-0.220$. The second linear piece of $g_{L}$ is then the tangent passing by $\psi_{2}$. These two tangents define two straight lines, which intersect at a point denoted $\psi_{12}=-0.154$. As this second straight line (tangent to $\psi_{2}$ ) deviates considerably from the exponentially growing curve $g$, we introduces a third tangent point $\psi_{3}$. This last point has been chosen in such a way as to minimize the area delimited by the curves $g$ and $g_{L}$. This is a simple criterion to minimize the error when we solve $\Delta \psi=g_{L}(\psi)$ instead of $\Delta \psi=g(\psi)$, in particular in the region $r \approx 0$ of the maximum of the stream function. With this choice we expect however, a difference in the value of the vorticity maximum of the observed and computed vortices $\left(g\left(\psi_{M}\right) \neq g_{L}\left(\psi_{M}\right)\right)$. We obtained the optimal value $-\psi_{3}=0.443$. The straight line passing by $\psi_{3}$ intersects the neighboring $\psi_{2}$ line at the $\psi_{23}$ point. The numerical values of the intersection points are $-\psi_{12}=0.154$ and $-\psi_{23}=0.345$ (circles in figure $6(\mathrm{~b})$ ).

The function $g_{L}$ is then linear in the intervals $I_{1}=$ $\left[\psi_{1}, \psi_{12}\right], I_{2}=\left[\psi_{12}, \psi_{23}\right]$, and $I_{3}=\left[\psi_{23}, \psi_{M}\right]$ (see the figure $6(\mathrm{~b})$ ). The images of these intervals in space, $J_{i}, i=$ $1,2,3$, define two matching points $R_{12}$, and $R_{23}<R_{12}$, such that $\psi\left(R_{12}\right)=\psi_{12}$ and $\psi\left(R_{23}\right)=\psi_{23}$. We have $J_{3}=\left[0, R_{23}\right], J_{2}=\left[R_{23}, R_{12}\right]$, and $J_{1}=\left[R_{12}, R\right]$, in increasing radius order.

Finally, it is also convenient to introduce the parameters $\kappa_{i}=\sqrt{\left|g^{\prime}\left(\psi_{i}\right)\right|}$, the inverses of the characteristic 
lengths associated with the slopes of $g(\psi)$ at $\psi=\psi_{i}$ for each $i\left(\kappa_{1}=0.548, \kappa_{2}=0.776, \kappa_{3}=1.826\right)$ and the constants $C_{i}=\psi_{i}-g\left(\psi_{i}\right) / g^{\prime}\left(\psi_{i}\right)$. They will be used in the analytic expression of the solution of (B2).

The next step is to obtain the radial symmetric solution $\psi=\psi(r)$, with $\psi(0)=\psi_{M}$, of the Helmholtz equations (B2), in a circular disc of radius $R$, where we should satisfy the boundary condition $\psi(R)=\psi_{1}=$ Const. These conditions together with the matching conditions of continuity and derivability at the interval limits, are in principle sufficient to obtain the stream function, and the vorticity distribution. However, in general, different solutions may be obtained that are compatible with these conditions, for example differing in the number of maxima. In order to select a solution having the same shape as the observed vortex, we note that $-\psi(r)$ is a decreasing function of $r$, and possesses only one maximum at $r=0$.

In each interval the solution of (B2) can be put as a superposition of (zero-order) Bessel functions. We consider first the central region of the vortex. Large positive values of $-\psi$ correspond to the interval $I_{3}$, where the general solution is

$$
\begin{gathered}
\psi(r)=A_{3} \mathrm{~J}_{0}\left(\kappa_{3} r\right)+B_{3} \mathrm{Y}_{0}\left(\kappa_{3} r\right)+C_{3}, \\
\psi \in I_{3}, 0 \leqslant r<R_{23},
\end{gathered}
$$

where $A_{i}$ and $B_{i}(i=3$ in this case), are constants to be determined by the matching conditions. As $\psi$ is smooth at the origin the coefficient $B_{3}$ of the second kind Bessel function $\mathrm{Y}_{0}$ must vanish. Moreover, $A_{3}$ must be negative in order to satisfy that $\psi$ is a monotonous increasing function. As $\psi$ has a single minimum (a maximum of $-\psi)$ at the origin, we have to satisfy $d \psi(0) / d r=0$.

When $r$ increases, $\psi$ enters in the interval $I_{2}$ where the solution is formally the same as in (C1), except for the value of the constants,

$$
\begin{gathered}
\psi(r)=A_{2} \mathrm{~J}_{0}\left(\kappa_{2} r\right)+B_{2} \mathrm{Y}_{0}\left(\kappa_{2} r\right)+C_{2}, \\
\psi \in I_{2}, R_{23}<r<R_{12} .
\end{gathered}
$$

According to the value of the coefficients $A_{2}$ and $B_{2}$ in $(\mathrm{C} 2),-\psi$ may strictly decrease and reach the interval $I_{1}$ or not. We assume the former case true, which, on one hand, avoid non physical solutions with multiple maxima due to the oscillatory nature of the Bessel functions $\mathrm{J}_{0}$ and $\mathrm{Y}_{0}$, and on the other hand, is compatible with the simulation where the boundary region of the vortex belongs to the interval $I_{1}$, where $-\psi<-\psi_{12}$.

In $I_{1}$, the positive slope of $g(\psi)$ gives solutions in terms of modified Bessel functions,

$$
\begin{gathered}
\psi(r)=A_{1} \mathrm{~K}_{0}\left(\kappa_{1} r\right)+B_{1} \mathrm{I}_{0}\left(\kappa_{1} r\right)+C_{1}, \\
\psi \in I_{1}, R_{12}<r<R .
\end{gathered}
$$

The first term in (C3) contains the modified Bessel function $\mathrm{K}_{0}$, which decays exponentially for large $r$. The behaviour of the vorticity tail will obviously depend on the actual value of the coefficients $A_{1}$ and $B_{1}$. However, one may anticipate that, as within this interval $\psi$ is small, the coefficient of the exponentially increasing $\mathrm{I}_{0}\left(\kappa_{1} r\right)$ Bessel function, must in fact be a rapidly decreasing function of the disc size $R$, which should insure the exponential decay of $\psi$. We confirmed this numerically. We see below, in a more general discussion on the properties of the thermodynamic states, that this property of exponential decay of the vortex at its boundary, is important to give a precise definition of a localized solution.

The unknown constants in (C1), (C2) and (C3), and the matching points $R_{12}$ and $R_{23}$, and the disc radius $R$, are determined using the the boundary conditions $\psi(R)=\psi_{2}$ and $\psi(0)=\psi_{M}$, together with the constraint on function $\psi(r)$ to be continue and derivable:

$$
\begin{gathered}
\psi_{12}=\left.\psi\left(R_{12}\right)\right|_{I_{1}}=\left.\psi\left(R_{12}\right)\right|_{I_{2}}, \\
\left.\frac{d}{d r} \psi\left(R_{12}\right)\right|_{I_{1}}=\left.\frac{d}{d r} \psi\left(R_{12}\right)\right|_{I_{2}}, \\
\psi_{23}=\left.\psi\left(R_{23}\right)\right|_{I_{2}}=\left.\psi\left(R_{23}\right)\right|_{I_{3}}, \\
\left.\frac{d}{d r} \psi\left(R_{23}\right)\right|_{I_{2}}=\left.\frac{d}{d r} \psi\left(R_{23}\right)\right|_{I_{3}} .
\end{gathered}
$$

Continuity of the vorticity is automatically satisfied by construction (definition of $g_{L}$ ). In fact, using the explicit form (C1)-(C3), one verifies that this set of conditions reduces to only two independent matching conditions. In general the number of independent equations is equal to the number of matching points $\left(R_{12}\right.$ and $R_{23}$ in the present case). Matching and boundary conditions constitute an optimization problem, which is numerically solved. The solution of the transcendental equations (B2), (C4) and (C5) gives the following values of the parameters: the constant coefficients, $A_{1}=-0.397$, $B_{1}=0.418, A_{2}=-0.435, B_{2}=0.210, A_{3}=-0.267$ and $B_{3}=0$; the matching points, $R_{23}=1.066$ and $R_{12}=1.797$; and the disc radius $R=2.375$. In figure 9 we show the scaled stream function and the vorticity.

Combining the solution (C1)-(C3) with the conditions (B3)-(B4), we can compute the fraction of positive vortices $n_{+}=0.602$ and the temperature parameter $\beta \Gamma^{2}=-651$. Recalling that we fixed the free parameter $\beta \Gamma$ equal to -0.301 , we can deduce $\beta=(\beta \Gamma)^{2} /\left(\beta \Gamma^{2}\right) \sim$ $-1.410^{-4}$.

\section{APPENDIX D: ABOUT THE CONVERGENCE OF THE ASYMPTOTIC METHOD}

In this Appendix we treat the problem of the convergence of the asymptotic method when the number of intervals $M$ increases. To the knowledge of the authors, there is no general mathematical results on the existence of a solution for quasi-linear elliptic operators of the form $\Delta \psi=g(\psi)$. It can nevertheless be proved in the cases where, in addition to some regularity properties, for $|\psi|$ large enough, $\psi g(\psi)$ is positive (Gilbarg \& Trudinger 1979). It can be easily checked that, in the present study, it involves problems with positive temperature (see (26)) and, therefore, does not support the existence of localized solutions. 


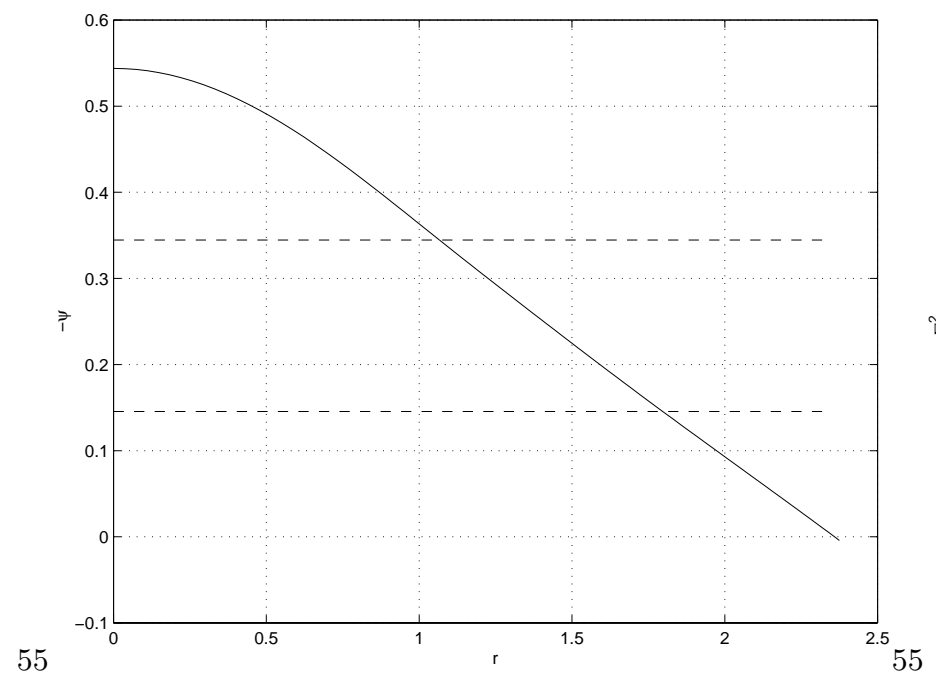

(a)

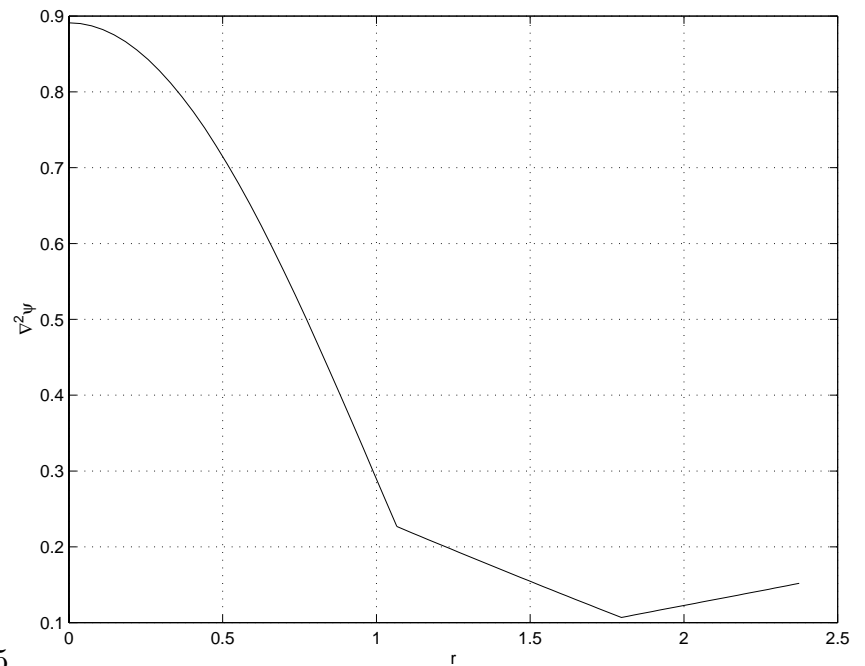

(b)

FIG. 9: Scaled stream function (a) and vorticity (b), solution of (B2) with temperature parameter $\beta \Gamma^{2}=-647$, and fraction of positive circulation $n_{+}=0.603$.

Note also that very few exact results exist on the convergence of the method we propose, based on a discretisation of right hand side $g$. Most of the results were instead obtained using a discretisation of the elliptic operator (the Laplacian in our case). In the following, we give an estimation of the speed of convergence of our method, under some assumptions connected to the existence of the solution.

For clarity, we denote the solution of the full initial problem $\psi_{N L}\left(\Delta \psi_{N L}=g\left(\psi_{N L}\right)\right)$ and the piecewise linear function $g_{L}, g_{L}^{M} . M$ is the number of points where $g_{L}^{M}$ and $g$ are equal and tangent. Let us define the space $\mathcal{C}$ of differentiable functions on the disk $C(R)$ of radius $R$ and center the axes origin and whose first derivatives are also continuous. The hypothesis are:

(i) For any $M(\geqslant 3)$ and a given set of numbers $\psi^{M}=\left\{\psi_{i}\right\}_{i=1}^{M}$, equation (B2) has a radial solution belonging to $\mathcal{C}, \psi=\psi_{M}(r)$ with $r \in[0, R]$.

(ii) There exists a finite positive number $\psi_{B}$ such that $\psi_{B}=\sup _{M} \sup _{r}\left|\psi^{M}(r)\right|<+\infty$.

Note that if $\psi_{M}$ converges uniformly towards a bounded function (which one expects to be $\psi_{N L}$ ), the second hypothesis is true. Moreover, we can always suppose that the intervals $I_{i}$ are included in $I_{B}=\left[-\psi_{B}, \psi_{B}\right]$ so as to be of finite length $\left(I_{i} \rightarrow I_{i} \cap I_{B}\right)$. Using a Taylor development, one can easily obtain the following majoration:

$$
\begin{gathered}
\sup \left\{\left|g(\psi)-g_{L}^{M}(\psi)\right|:|\psi| \leqslant \psi_{B}\right\} \mid \leqslant \\
\frac{1}{2} \max _{i} \delta_{i, M}^{2} \max \left\{\left|g^{(2)}(\psi)\right|:|\psi| \leqslant \psi_{B}\right\},
\end{gathered}
$$

where $\delta_{i, M}$ denotes the length of the interval $I_{i}$ at the order $M$. Using the second hypothesis stated above, the last inequality gives

$$
\sup _{r \in[0, R]}\left|\Delta \psi_{M}(r)-g_{N L}\left(\psi_{M}(r)\right)\right|=0\left(1 / M^{2}\right),
$$

which shows the convergence of the algorithm when $M$ is going to the infinity. Note that (D2) does not imply, a priori, that the set of solutions $\psi_{M}$ converge towards $\psi_{N L}$ in any sense. Probably, we have to take into account some specific properties of the functions $\psi_{M}$ and $g_{L}^{M}$ (as for instance the fact that the $\psi_{M}(r)$ should be decreasing functions of $r$ ) to show the convergence of $\psi_{M}$. It would be interesting to get some general results for this type of problems.

Nevertheless, it is easy to show that if $\psi_{M}$ converges to a function $\psi_{\infty}$ in space $\mathcal{C}$ (which means uniform convergence of $\psi_{M}$ to $\psi_{\infty}$ and of $\nabla \psi_{M}$ to $\left.\nabla \psi_{\infty}\right)$, then $\psi_{\infty}$ is a weak solution of the initial problem (as we show below). By definition (Gilbarg \& Trudinger 1979), $\psi_{\infty}$ is a weak solution of the initial problem if $\psi_{\infty} \in \mathcal{C}$ and

$$
\int_{C(R)}\left[\nabla \psi_{\infty}(\mathbf{r}) \nabla f(\mathbf{r})-g\left(\psi_{\infty}(\mathbf{r})\right) f(\mathbf{r})\right] d \mathbf{r}=0
$$

for all $f \in \mathcal{C}$ such that $f(R)=0$. If $\psi_{M}$ converges to a function $\psi_{\infty} \in \mathcal{C}$, then from the inequality

$$
\left|g_{L}^{M}\left(\psi_{M}\right)-g\left(\psi_{\infty}\right)\right| \leqslant\left|g_{L}^{M}\left(\psi_{M}\right)-g\left(\psi_{M}\right)\right|+\left|g\left(\psi_{M}\right)-g\left(\psi_{\infty}\right)\right|,
$$

and the uniform convergence on $I_{B}$ of $g_{L}^{M}$ to $g$ (by construction), it follows that $g_{L}^{M}\left(\psi_{M}\right)$ converges uniformly to $g\left(\psi_{\infty}\right)$. Moreover we have for any integer $M$, the relation

$$
\begin{aligned}
& \left|\int_{\mathcal{D}}\left[\nabla \psi_{\infty} \nabla f-g\left(\psi_{\infty}\right)\right] d \mathbf{r}\right|= \\
& \quad \int_{\mathcal{D}}\left\{\nabla\left(\psi_{\infty}-\psi_{M}\right) \nabla f+\left[g_{L}^{N}\left(\psi_{M}\right)-g\left(\psi_{\infty}\right)\right] f\right\} d \mathbf{r} \\
& \leqslant \sup _{r}\left|\nabla \psi_{M}-\nabla \psi_{\infty}\right| \int_{C(R)}|\nabla f| d \mathbf{r}+ \\
& \quad \sup _{r}\left|g_{L}^{M}\left(\psi_{M}\right)-g\left(\psi_{\infty}\right)\right| \int_{C(R)}|f| d \mathbf{r}
\end{aligned}
$$


The term on the right hand side converges to zero with $M \rightarrow+\infty$, it follows that (D3) is true and therefore $\psi_{\infty}=\psi_{N L}$, in the sense that this is a weak solution of the problem. As a final remark, note also that conver- gence of $\psi_{M}$ implies the convergence of the vorticity and temperature conditions (B3, B4) to the ones of the initial problem $(27,28)$.
[1] Bartello, P. and Warn, T. Self-similarity of decaying twodimensional turbulence. J. Fluid Mech. 326, 357 (1996).

[2] Berdichevsky, V. L. Statistical mechanics of point vortices. Phys. Rev. E 51, 4432 (1995).

[3] Brands, H., Stulemeyer, J., Pasmanter, R. A. and Schep, T. J. A mean field prediction of the asymptotic state of decaying 2D turbulence. Phys. Fluids 9, 2815 (1997).

[4] Bracco, A., McWilliams, J. C., Murante, G., Provenzale, A. and Weiss, J. B. Revisiting freely decaying turbulence at millennial resolution Phys. Fluids 12, 2931 (2000).

[5] Carnevale, G. F., McWilliams, J. C., Pomeau, Y., Weiss, J. B. and Young, W. R. Rates, pathways, and end states of nonlinear evolution in decaying twodimensional turbulence: Sacaling theory versus selective decay. Phys. Fluids A 4, 1314 (1992).

[6] Chavanis, P.-H. From Jupiter's great red spot to the structure of galaxies: Statistical mechanics of $2 \mathrm{D}$ vortices and stellar systems. Ann. N. Y. Acad. Sci. 867, 120 (1998).

[7] Chavanis, P.-H. and Somméria, J. Clasification of selforganized vortices in two-dimensional turbulence: the case of a bounded domain. J. Fluid Mech. 314, 267 (1996).

[8] Chavanis, P.-H. and Sommeria, J. Thermodynamical approach for small-scale parametrization in 2D turbulence. Phys. Rev. Lett. 78, 3302 (1997).

[9] Chavanis, P.-H. and Sommeria, J. Clasification of robust isolated vortices in two-dimensional hydrodynamics. $J$. Fluid Mech. 356, 259 (1998).

[10] Chavanis, P.-H. and Sommeria, J. Statistical mechanics of the shallow water system Phys. Rev. E 65, 026302 (2002).

[11] Couder, Y. Observations expérimentales de la turbulence bidimensionnelle dans un film liquide mince C. R. Acad. Sc. Paris II 297,641 (1983).

[12] Ferro Fontán, C. and Verga, A. D. Dynamics of coherent structures and turbulence of plasma drift waves. Phys. Rev. E 52, 6717-6735.

[13] Fine, K. S., Kass, A. C., Flynn, W. G. and Driscoll, C. F. Relaxation of $2 \mathrm{D}$ turbulence to vortex crystals Phys. Rev. Lett. 75, 3277 (1995).

[14] Gazdag, J. 1976 Time-differencing schemes and transform methods. J. Comp. Phys. 20, 196 (1995).

[15] D. Gilbarg and N. S. Trudinger Elliptic Partial Differential Equations of Second Order. (Springer, Berlin, 1977), (see Theorem 14.10 together with condition 9.30 and definition 10.19).

[16] G. Haller and G. Yuan Lagrangian coherent structures and mixing in two-dimensional turbulence. Physica $D$ 147, 352 (2000).

[17] Hasegawa, A. Self-organization processes in continuous media. Adv. Phys. 34, 1 (1985).

[18] Hasegawa, A., Maclennan, C. G. and Kodama, Y. Nonlinear behavior and turbulence spectra of drift waves and Rossby waves. Phys. Fluids 22, 2122 (1979).
[19] Hopfinger, E. J. and van Heijst, G. J. F. Vortices in rotating fluids Ann. Rev. Fluid Mech. 25, 241 (1992).

[20] Jiménez, J. Hyperviscous vortices. J. Fluid Mech. 279, 169 (1994).

[21] Jin, D., Z. and Dubin, H., E. Regional Maximum Entropy Theory of vortex Crystal Formation. Phys. Rev. Lett. 80, 4434 (1998).

[22] Kraichnan, R. H. Inertial Ranges in Two-Dimensional Turbulence. Phys. Fluids 10, 1417 (1967).

[23] Joyce, G. and Montgomery, D. Negative temperature states for the two-dimensional guiding-centre plasma. J. Plasma Phys. 10, 107 (1973).

[24] Lundgren, T. S. and Pointin, Y. B. Non-Gaussian probability distributions for a vortex fluid. Phys. Fluids 20, 356 (1977).

[25] Marchioro, C. and Pulvirenti, M Mathematical Theory of Incompressible Nonviscous Fluids. (Springer, Berlin, 1994).

[26] Marcus, P. S. Numerical simulation of Jupiter's great red spot Nature 331, 693 (1988).

[27] Matthaeus, W. H., Stribling, W. T., Martinez, D., Oughton, S. and Montgomery, D. Decaying, twodimensional, Navier-Stokes turbulence at very long times. Physica D 51, 531 (1991).

[28] McWilliams, J. C. The emergence of isolated coherent vortices in turbulent flow. J. Fluid Mech. 146, 21 (1984).

[29] McWilliams, J. C. The vortices of two-dimensional turbulence. J. Fluid Mech. 219, 361 (1990).

[30] Miller, J., Weichman, P. B. and Cross, M. C. Statistical mechanics, Euler's equation, and Jupiter's Red Spot. Phys. Rev. A 45, 2328 (1992).

[31] Montgomery, D., Matthaeus, W. H., Stribling, W. T., Martinez and D., Oughton, S. Relaxation in two dimensions and the "sinh-Poisson" equation Phys. Fluids A 4, 3 (1992).

[32] Nezlin, M. V. and Chernikov, G. P. Analogy between drift vortices in plasma and geophysicql hydrodynqmics. Plasma Phys. Reports 21, 975 (1995).

[33] Novikov, E. A. Stochastization and collapse of vortex systems. Annals NY Acad. Sci. 357, 47 (1980).

[34] Onsager, L. Statistical hydrodynamics. Nuovo Cimento Suppl. 6, 279 (1949).

[35] Pésceli, H. L. and Trulsen, J. Eulerian and Lagrangian velocity correlations in two-dimensional random geostrophic flows. J. Fluid Mech. 338, 249 (1997).

[36] Pointin, Y. B. and Lundgren, T. S. Statistical mechanics of two-dimensional vortices in a bounded container. Phys. Fluids 19, 1459 (1976).

[37] Robert, R. and Sommeria, J. Statistical equilibrium states for two-dimensional flows. J. Fluid Mech. 229, 291 (1991).

[38] Santangelo, P., Benzi, R. and Legras, B. The generation of vortices in high-resolution, two-dimensional decaying turbulence and the influence of initial conditions on the breaking of self-similarity. Phys. Fluids A 6,1027 (1989). 
[39] Segre, E. and Kida, S. Late states of incompressible 2D decaying vorticity flields. Fluid Dynamics Research 23, 89 (1998).

[40] Sommeria, L., Nore, C., Dumont, T. and Robert, R. Théorie statistique de la tache rouge de Jupiter. C. $R$. Acad. Sci. Paris, Série II 312, 999 (1991).

[41] Sommeria, J., Staquet, C. and Robert, R. Final equilibrium state of a two-dimensional shear layer. J. Fluid Mech. 233, 661 (1991.

[42] Stewart, H. J. Periodic properties of the semi-permanent atmospheric pressure systems. Q. Appl. Math. 1, 262 (1943.

[43] Taylor, M. E. Partial Differential Equations III. Nonlinear Equations. (Springer, Berlin, 1996).

[44] Ting, A. C., Chen, H. H. and Lee, Y. C. Exact solutions of a nonlinear boundary value problem: the vortices of the two-dimensional sinh-Poisson equation. Physica D 26, 37 (1987).

[45] Weiss, J. W. and McWilliams, J. C. Nonergodicity of point vortices Phys. Fluids A 3, 835 (1991).

[46] Weiss, J. W. and McWilliams, J. C. Temporal scaling behavior of decaying two-dimensional turbulence Phys. Fluids A 5, 608 (1993).

[47] Watanabe, T., Iwayama, T. and Fujisaka, H. Scaling law for coherent vortices in decaying drift Rossby wave turbulence Phys. Rev. E 57, 1636 (1998).

[48] Yin, Z., Montgomery, D. C. and H. J. H. Clercx, Alternative statistical-mechanical descriptions of dacaying two-dimensional turbulence in terms of "patches" and "points", preprint arXiv:physics/0211024 (2002).

[49] See Appendix C and D online (PAPS). 